\title{
Regulation of lateral root development by shoot-sensed far-red light via HY5 is nitrate-dependent and involves the NRT2.1 nitrate transporter.
}

$1 \quad$ Kasper van Gelderen ${ }^{1 *}$, Chiakai Kang ${ }^{1}$, Peijin $\mathrm{Li}^{1}$ and Ronald Pierik ${ }^{1 *}$

2 Plant Ecophysiology, Science faculty, Biology department, Utrecht University, Padualaan 8,

$33584 \mathrm{CH}$, Utrecht, The Netherlands

4 * Correspondence:

5 Corresponding Authors

6 k.vangelderen@uu.nl

$7 \quad$ r.pierik@uu.nl

9 Keywords: Shade avoidance, Far-Red light, Nitrate signaling, Nitrate deficiency, Root plasticity, D-root, HY5, NRT2.1, Photobiology.

\section{Abstract}

12 Plants are very effective in responding to environmental changes during competition for light and

13 nutrients. Low Red:Far-Red (low R:FR)-mediated neighbor detection allows plants to compete

14 successfully with other plants for available light. This above-ground signal can also reduce lateral

15 root growth by inhibiting lateral root emergence, a process that might help the plant invest resources

16 in shoot growth. Nitrate is an essential nutrient for plant growth and Arabidopsis thaliana responds to

17 low nitrate conditions by enhancing nutrient uptake and reducing lateral and main root growth. There

18 are indications that low R:FR signaling and low nitrate signaling can affect each other. It is unknown

19 which response is prioritized when low R:FR light- and low nitrate signaling co-occur. We

20 investigated the effect of low nitrate conditions on the low R:FR response of the A. thaliana root

21 system in agar plate media, combined with the application of supplemental Far-Red (FR) light to the

22 shoot. We observed that under low nitrate conditions main and lateral root growth was reduced, but

23 more importantly, that the response of the root system to low R:FR was suppressed. Consistently, a

24 loss-of-function mutant of a nitrate transporter gene NRT2.1 lacked low R:FR-induced lateral root 
reduction and its root growth was hypersensitive to low nitrate. ELONGATED HYPOCOTYL5

(HY5) plays an important role in the root response to low R:FR and we found that it was less

sensitive to low nitrate conditions with regards to lateral root growth. In addition, we found that low

R:FR increases NRT2.1 expression and that low nitrate enhances HY5 expression. HY5 also affects

NRT2.1 expression, however, it depended on the presence of ammonium in which direction this

effect was. Replacing part of the nitrogen source with ammonium also removed the effect of low

R:FR on the root system, showing that changes in nitrogen sources can be crucial for root plasticity.

Together our results show that nitrate signaling can repress low R:FR responses and that this involves signaling via HY5 and NRT2.1.

\section{Introduction}

Plants adapt their growth and development to compete for the limited light and nutrients with which they grow their bodies. Plant can sense competing neighbours via Far-Red (FR) light that is reflected by leaves of neighbouring plants. This reflection of FR light leads to a lowering of the Red to FarRed ratio (R:FR). Plants respond to this low R:FR by elongating their aboveground organs in an effort to reach for the sunlight. This adaptive response to future competition is what we call the shade avoidance response (Ballaré and Pierik, 2017). The R:FR ratio is sensed by Phytochrome photoreceptors; Phytochromes are activated by R light, changing them to active Pfr state and inactivated by FR light which changes them back to inactive Pr state. The active Pfr form of phytochromes phosphorylates and interacts with PHYTOCHROME INTERACTING FACTORS transcription factors that regulate light and temperature responses (Leivar and Monte, 2014).

Plant roots are essential for the uptake of water and nutrients from the soil, but root growth is impossible without sugars supplied from the shoot. This interdependency between root and shoot means that signaling between these organs is essential to achieve optimal growth (Van Gelderen et al., 2018). The root system responds to low R:FR-mediated plant competition via by reducing its growth (Salisbury et al., 2007; van Gelderen et al., 2018). Normally the root system cannot directly detect the above-ground low R:FR ratio, therefore a mobile, FR-induced, bZip transcription factor

53 ELONGHATED HYPOCOTYL 5 (HY5) travels from shoot to root to affect root growth

54 belowground (Chen et al., 2016; van Gelderen et al., 2018). In the root, HY5 increases its own 
expression (Zhang et al., 2017) and represses auxin signaling and lateral root development (Sibout et al., 2006; Cluis et al., 2004). The current model is that FR light enhances HY5 transport to the root, which leads to repression of lateral root emergence by repressing auxin signaling and transport around the developing lateral root primordium (LRP) (van Gelderen et al., 2018).

HY5 transport can also affect nutrient uptake by upregulating transcription of the nitrate transporter gene NRT2.1 (Chen et al., 2016; Jonassen et al., 2008, 2009). Nitrate is a crucial resource for plant life which is taken up by the root and transported through the xylem to the shoot. There are several transmembrane nitrate transporters that facilitate this uptake. NRT1.1 is a transporter/receptor that plays a crucial role in constant high-affinity nitrate uptake, when nitrate is sufficient (Krouk et al., 2006). NRT2.1 is an important high affinity nitrate transporter in the root (Cerezo et al., 2001) that is upregulated when nitrate concentrations are low and NRT2.1 is crucial for low nitrate responses (O'Brien et al., 2016). Another way that shoot-derived HY5 can regulate nutrient uptake is by upregulating the transcription of the phosphate transporter gene $P H T 1$, much in the same manner as in the case of NRT2.1 (Sakuraba et al., 2018). Therefore, it is clear that shoot-perceived low R:FR could regulate nutrient uptake via the root through shoot-to-root transport of HY5.Thus, if light quality can influence nutrient uptake-associated transporters, can nutrient signaling affect low R:FRmediated changes in root development? In order to test this hypothesis, we grew Arabidopsis thaliana in the D-root petri-plate system that allows roots to be kept in darkness despite the plant being on an agar plate (Silva-Navas et al., 2015). In this way only the shoot, and not the root, is experiencing a low R:FR ratio, which we achieve by the addition of supplemental FR to the white light background (WL+FR)(van Gelderen et al., 2018). We combined this setup with different nitrate-containing media and observed that low nitrate inhibited the response of the root and shoot to shoot-perceived WL+FR. Through mutant analyses we were able to show that in addition to HY5, NRT2.1 is also involved in WL+FR-mediated root growth reduction. qRT-PCR Expression analysis showed that both WL+FR light and low nitrate induce NRT2.1 expression. Additionally, low nitrate induced expression of HY5, which linked changes in NRT2.1 and HY5 expression and lateral root development phenotypes. Interestingly, the role of HY5 in regulating NRT2.1 expression was highly

82 dependent on the nitrogen source used (ammonium and/or nitrate). Together these results provide a

83 causal link for the integration of WL+FR signaling from the shoot with nutrient signaling in the root via HY5 and NRT2.1. 


\subsection{Plant material}

87 In all experiments Columbia-0 seeds were used as wild type. Mutants used that were previously 88 described are: hy5-2 hyh (van Gelderen et al., 2018; Zhang et al., 2017), hy5-215 (Oyama et al., 89 1997), nrt2.1 nrt2.2 (Li et al., 2007) and chl1-5 (Mounier et al., 2014).

\section{$90 \quad 2.2 \quad$ Growth conditions}

91 Plants were grown on either $1 / 2 \mathrm{MS}$ medium with addition of $1 \mathrm{~g} / \mathrm{l} \mathrm{MES}$ and $\mathrm{pH}$ of 5.8 with $0.8 \%$ 92 plant agar (Duchefa), or modified versions of the medium described in Kellermeier et al., 2014

93 (Table 1), also with the addition of MES and agar. The inserts of the D-root system combined with 94 black paper covers were used to shield the roots from light (Silva-Navas et al., 2015) and the plates 95 were sealed with urgopore tape. The light regime was 16 hours light, 8 hours dark. Photosynthetically 96 Active Radiation (PAR) was $140 \mu \mathrm{mol} / \mathrm{m}^{2} / \mathrm{s}$ (Philips HPI $400 \mathrm{~W}$ ), FR light was added using Philips

97 GreenPower LED research modules, far red, $24 \mathrm{Vdc} / 10 \mathrm{~W}, 730-\mathrm{nm}$ peak, emitting $\sim 25 \mu \mathrm{mol} / \mathrm{m}^{2} / \mathrm{s} \mathrm{FR}$ 98 light at $20 \mathrm{~cm}$ distance. The LEDs were placed at $9 \mathrm{~cm}$ height, facing the plates sideways.

99 Temperature was $20^{\circ} \mathrm{C}$ and humidity $70 \%$. Seeds were surface sterilized using chlorine gas

100 (bleach $+\mathrm{HCl}$ ) for two hours and aerated in a flow cabinet for 15 minutes. Sterilised seeds were sown 101 on one row at $9 \mathrm{~cm}$ height with 27 seeds on one $12 \mathrm{~cm}$ square Greiner petri dishes containing agar 102 medium and were the sealed and vernalized at $4{ }^{\circ} \mathrm{C}$ for 3-6 days. For growth, plates were placed in 103 white light (WL) first and after one day of germination were placed in either WL or WL+FR. After 104 four days seedlings were transferred from starting plates to new identical plates, but with 5 seedlings 105 per plate. At 8-9 days plates were scanned.

\section{$106 \quad 2.3 \quad$ Image acquisition, root phenotyping and data processing}

107 Plates were scanned using an Epson V850 flatbed photonegative scanner at 1200 dpi. Hypocotyl

108 length was analyzed with standard ImageJ tools. Root phenotyping was performed using Smartroot 109 (Lobet et al., 2011). Data was processed with R and statistical analysis was performed with both $\mathrm{R}$ 110 and Prism.

\section{$111 \quad 2.4$ Seedling fixation and lateral root primordia analysis.}

112 After scanning, seedlings were fixed according to the protocol of Malamy and Benfey, 1997a.

113 Seedlings were mounted in 50\% glycerol and slides were sealed with nail polish. Slides were 
114 analyzed using a Zeiss Axioskop2 DIC (differential interference contrast) microscope (40X Plan-

115 NEOFLUAR DIC objective) with a Lumenera Infinity1 camera.

\section{$116 \quad 2.5 \quad$ RNA extraction and qRT-PCR expression analysis}

117 For gene expression analyses, plants were sown at 16 seeds in a row and kept in the growth

118 conditions mentioned above for 5 days. Between 15 to 19 seedlings were harvested per sample, only

119 root tissues were used for RNA extraction. Four biological replicates were taken per

120 treatment/genotype condition. The Qiagen plant RNeasy kit was used for RNA extraction. First-

121 strand cDNA was made using the Thermo Scientific RevertAid H Minus Reverse Transcriptase,

122 RiboLock RNase inhibitor, and Invitrogen random hexamer primers. RNA input into the cDNA

123 reaction was kept equal within experiments. Primers were designed preferably across introns and for

124 100- to 150-bp fragments with an annealing temperature of $60^{\circ} \mathrm{C}$ with primer3plus (http://www.

125 bioinformatics.nl/cgi-bin/primer3plus/primer3plus.cgi). Primers were tested for efficiency using

126 generic Col-0 cDNA at a concentration range of $2.5 \rightarrow 40 \mathrm{ng}$ of cDNA per $5 \mathrm{~mL}$ reaction. qPCR

127 reagents used were Bio-Rad SYBR-Green Mastermix on 384-well plates in a Life Technologies

128 ViiA7 real-time PCR system. All CT values were normalized against two validated housekeeping

129 genes: ADENINE PHOSPHORIBOSYL TRANSFERASE1 (APT1) and PROTEIN PHOSPHATASE $2 A$

130 SUBUNIT A3 (PP2AA3). The DDCT method was used to calculate relative expression values (Livak 131 and Schmittgen, 2001). Primer sequences are provided in Table 2.

\section{$\begin{array}{lll}132 & 3 & \text { Results }\end{array}$}

\section{Low Nitrate decreases the response to WL+FR in both the hypocotyl and root}

134 In order to investigate the effect of low nitrate on the root system of Arabidopsis thaliana, we used a

135 growth medium with mineral composition as published in (Kellermeier et al., 2014)(Table 1).

136 Ammonium was left out to remove any interfering effects with nitrate signaling (Hachiya and

137 Sakakibara, 2017). Since our previous work (van Gelderen et al., 2018) on the response of the root

138 system to WL+FR was based on plates containing $1 \frac{2}{2}$ MS we compared the nitrate-only-N medium

139 and $1 / 2$ MS media with respect to wildtype Col-0 responses to WL+FR. We employed the use of the

140 D-root system (Silva-Navas et al., 2015), in order to grow the root system under physiologically

141 meaningful conditions that avoid light exposure (van Gelderen et al., 2018). Overall, Col-0 wild type

142 responded in a similar manner to $\mathrm{WL}+\mathrm{FR}$ on the nitrate-only-N medium compared to when these

143 plants were grown 1/2 MS-containing medium (Figure 1A-D). WL+FR stimulated hypocotyl length 
144 (Figure 1A), whereas lateral root density and main root length were reduced (Figure 1B-C). Having

145 confirmed that the nitrate-only-N medium gave similar FR-induced root and shoot architecture

146 phenotypes as on $1 / 2 \mathrm{MS}$ we proceeded to investigate the low nitrate response. A ten-fold lower

147 concentration $(0.2 \mathrm{mM})$ compared to control $(2 \mathrm{mM})$ of nitrate resulted in a reduction of hypocotyl

148 elongation in WL+FR when compared to $2 \mathrm{mM}$ nitrate (Figure 1E,H). The reduction of lateral root

149 density and main root length due to WL+FR was lost in the low nitrate condition (Figure 1 F,G,H).

150 These results indicate that a low nitrate medium leads to the loss or reduction of WL+FR-induced

151 changes of root and shoot development. We verified if this is a nitrogen-specific effect, by

152 performing a comparable experiment, but now depleting phosphate. Whereas similarly to low nitrate

153 hypocotyl length was reduced, lateral root density was increased by low phosphate (Figure 1E,F,H),

154 rather than decreased as in low nitrate. Interestingly, WL+FR did not decrease the lateral root density

155 in low phosphate, however it did decrease the main root length (Figure 1F,G). These results show

156 that the specific nutrient status of the medium and/or the plant affects the manner in which root

157 system development integrates with the light spectral composition to which the shoot is exposed.

\subsection{NRT2.1/2.2 and HY5 HYH are required for the combined WL+FR and nitrate response}

160 Previous work identified that root development of the hy5 hyh double mutant is unresponsive to

161 WL+FR (van Gelderen et al., 2018). Furthermore, HY5 regulates nitrate uptake via the

162 transcriptional control of the nitrate transporter gene NRT2.1 (Chen et al., 2016; Jonassen et al., 2009,

163 2008). NRT2.1 is part of the high affinity nitrate uptake pathway and NRT2.1 inhibits the initiation

164 of lateral root primordia during nitrate starvation (Remans et al., 2006; Little et al., 2005). Therefore,

165 we tested both hy5 hyh and nrt2.1 nrt2.2 mutants in WL or WL+FR conditions on normal and low

166 nitrate media (a single T-DNA knocks out both $n r t 2.1$ and 2.2 (Li et al., 2007)). WL+FR again led to

167 a decrease in main root length in Col-0, which was abolished in low nitrate (Figure 2A,C). Both

$168 n r t 2.1$ nrt2.2 and hy5 hyh lacked the WL+FR-induced main root length decrease, but did show a

169 strong main root length reduction upon exposure to low nitrate (Figure 2A,D,E). Col-0 lateral root

170 density on normal nitrate was reduced by WL+FR, however this did not occur in $n r t 2.1$ nrt2.2

171 (Figure 2B), while low nitrate strongly reduced nrt2.1 nrt2.2 lateral root density (Figure 2B). Even

172 more striking was the fact that the lateral root density of hy5 hyh did not significantly change in any

173 of the conditions (Figure 2B). The hypocotyl elongation responses to WL+FR of both $n r t 2.1$ nrt2.2

174 and hy5 hyh mutants were similar in trend to Col-0, however nrt2.1 nrt2.2 was slightly more 


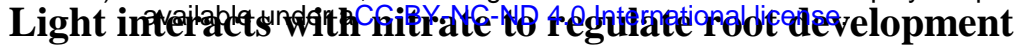

175

176

177

178

179

180

181

182

183

184

185

186

187

188

189

190

191

192

193

194

195

196

197

198

199

200

201

202

203

204

205

sensitive to low nitrate and hy5 hyh has a much longer hypocotyl length to start out. (Figure S1A). To test the limits of nitrate depletion further we grew the same mutants on a lower concentration of nitrate $(0.05 \mathrm{mM})$. Both Col-0 and $n r t 2.1 \mathrm{nrt2} .2$ lateral root density were severely reduced by this depletion, however the lateral root density of hy5 hyh was only slightly affected (Figure S1B). These results confirm the enhanced sensitivity of $n r t 2.1$ nrt 2.2 to low nitrate conditions, but also indicate a reduced sensitivity of the hy5 hyh mutant to low nitrate. Furthermore, both mutants lacked the lateral root density response to $\mathrm{WL}+\mathrm{FR}$, showing that it is likely they are both involved in mediating the response to $\mathrm{WL}+\mathrm{FR}$.

\subsection{Low nitrate medium affects lateral root primordia development.}

The results presented here and from various other works show that lateral root growth is affected by low nitrate conditions (Kellermeier et al., 2014; Gruber et al., 2013; Bouguyon et al., 2016). It is also known that in certain conditions NRT2.1 can inhibit lateral root initiation (Little et al., 2005; Remans et al., 2006). To get more insight into what developmentally occurs with lateral root primordia (LRP) growth - whether there is a decrease in initiation, emergence, or a mid-development arrest - we analyzed the seedlings shown in Figure 2 for the frequency of lateral root primordia stages, according to the classification of Malamy and Benfey, 1997 (Figure 3). Col-0 seedlings exposed to WL+FR and grown on $2 \mathrm{mM}$ nitrate had an increase in stage 1+2 and stage 5+6 lateral root primordia when compared to WL-grown seedlings, while the emerged primordia $(7+\mathrm{E})$ were decreased (Figure $3 \mathrm{~A})$. This result was similar to a previously published experiment performed on $1 / 2 \mathrm{MS}$-containing plates (van Gelderen et al., 2018) and indicates that primordia are formed, but do not fully develop into lateral roots. Col-0 seedlings on low nitrate $(0.2 \mathrm{mM})$ plates had no LRP stage frequency differences between WL and WL+FR (Figure 3A). Strikingly, nrt2.1 nrt2.2 mutant seedlings did not have any significant differences between treatments, indicating that both the effect of low nitrate and WL+FR on LRP development are dependent upon nrt2.1 nrt2.2 (Figure 3B). The hy5 hyh LRP stages did have significant differences, which overall appeared to be opposite to those in Col-0. In the hy5 hyh genotype, WL+FR led to more 7+E stages in normal and low nitrate, while LRP stage 1+2 and 3+4 frequency was reduced (Figure 3C). However, the effect of low nitrate on hy5 hyh LRP stages was relatively minor, when compared to Col-0. It is surprising that there were significant changes in late lateral root primordia stages in hy5 hyh, since the lateral root density hardly changed between the four conditions (Figure 2C). In hy5 hyh there was not a significant difference in the total number of 
primordia between WL and WL+FR (Figure S2), thus it is possible that these extra primordia have not yet resulted in a visually changed lateral root outgrowth. Overall, these results show that low nitrate removes any WL+FR effect on lateral root primordia in Col-0 and that $n r t 2.1$ nrt2.2 is unresponsive to both low nitrate and WL+FR, while hy5 hyh has a distinctly different response than Col-0.

\subsection{HY5 and WL+FR regulate NRT2.1 expression}

HY5 is able to promote transcription of NRT2.1 and it is also upregulated by low nitrate levels (Chen et al., 2016; Okamoto et al., 2003). Since HY5 is crucial for the root response to shoot-perceived

214 WL+FR (Figure 2), we were interested in investigating if WL+FR can also affect NRT2.1 expression. We performed a time-course qRT-PCR experiment seedling root material during the 16 hours photoperiod to map when the response of HY5 and NRT2.1 to WL+FR was strongest (Figure 4A,B). We observed that during the day NRT2.1 expression kept rising and that, except at 12 hours postdawn, WL+FR led to an additional increase in NRT2.1 expression (Figure 4A). HY5 expression peaked at 4 and 8 hours into the day, at which time it was also upregulated by WL+FR (Figure 4B). Next, we grew Col-0 and hy5 hyh seedlings on normal or low nitrate plates, and in WL or WL+FR. NRT2.1 was upregulated in Col-0 WL+FR treated seedlings (Figure 4C). We did not observe the expected strong increase of NRT2.1 transcription due to the low nitrate levels, however that can be explained by the duration of the low nitrate treatment (5 days), since upregulation of NRT2.1 by low nitrate is a transient effect and decreases slowly after 1 day (Okamoto et al., 2003). Importantly, in the hy5 hyh mutant background NRT2.1 transcription was upregulated and the increase in WL+FR was absent, however the increase due to low nitrate was still present (Figure 4C). The observed elevated expression of NRT2.1 in the hy5 hyh mutant is in contrast with the previously shown stimulation of this gene by HY5 (Chen et al., 2016). We performed a similar experiment with another hy5 mutant, hy5-215, on normal nitrate medium, and the result was the same (Figure S3A). However, we observed that when we grew hy5-215 on 1/2 MS medium, a strong decrease of NRT2.1 expression was observed (Figure S3B). Therefore, we hypothesized that the addition of ammonium might be a crucial element in regulating NRT2.1 expression through HY5. To test this, we made a medium with $2 \mathrm{mM}$ consisting of $1.33 \mathrm{mM} \mathrm{NO}_{3}{ }^{-}$and $0.67 \mathrm{mM} \mathrm{NH}_{4}{ }^{+}$, a nitrate/ammonium ratio that is very similar to $1 / 2$ MS. When we repeated the experiment with the addition of this combined nitrateammonium we observed a strong decrease in NRT2.1 expression on the combined nitrate-ammonium medium, both in wild type, and hy5 hyh (Figure 4D). Again, we observed an increase in NRT2.1 
238 there was no increase in the WL+FR \& low nitrate condition. In addition, we tested the expression of

239 the close homologue NRT2.2, which displayed a strong response to WL+FR and a very strong

240 response to low nitrate (Figure 4E). Its expression was higher in the hy5 hyh background, however in

241 this mutant NRT2.2 did still respond to WL+FR and low nitrate, indicating that NRT2.2 is regulated

242 in a somewhat different manner than NRT2.1. NRT2.2 expression was decreased in the combined

243 nitrate-ammonium medium to almost undetectable levels (Figure 4E). We tested the expression of

244 HY5 on the same material and observed an increase in expression due to WL+FR and interestingly,

245 also an increase due to low nitrate (Figure 4F). In both low nitrate and combined nitrate-ammonium

246 medium the expression of HY5 did not change due to WL+FR (Figure 4F), while HY5 expression

247 was not changed in the $n r t 2.1$ nrt2.2 mutant (Figure 4G). These results show that shoot-perceived FR

248 light and low nitrate leads to increased expression of NRT2.1, NRT2.2 and HY5. However, in low

249 nitrate the upregulation of these genes due to shoot-perceived FR is less. In the hy5 hyh mutant,

$250 N R T 2.1$ and NRT2.2 are upregulated, indicating a negative effect of HY5 on their transcription,

251 however this effect can be fully masked by the addition of ammonium. Importantly, in the nrt2.1

252 nrt2.2 mutant, HY5 expression is not changed, indicating that NRT2.1 likely acts downstream, and

253 not upstream, of HY5.

\subsection{Ammonium addition masks WL+FR effect on root development}

Addition of ammonium as a nitrogen source led to a very strong decrease in NRT2.1 and NRT2.2

expression. This prompted us to investigate if the addition of ammonium had significant effects on the root developmental response to WL+FR. Ammonium can stimulate lateral root initiation and directly promote lateral root emergence, while ammonium-dependent signaling can decrease part of the primary low nitrate response (Hachiya and Sakakibara, 2017; Lima et al., 2010; Meier et al., 2020). The addition of ammonium had little effect on hypocotyl elongation (Figure S4A). Interestingly, the addition of ammonium led to the loss of difference in lateral root density between WL and WL+FR as seen in Col-0 on nitrate-only-N medium (Figure 5A,C). On nitrate-only-N medium, the $n r t 2.1$ nrt2.2 mutant had a reduced lateral root density compared to Col-0 WL, but the addition of ammonium removed this effect and led to increased lateral root density (Figure 5A). On the combined ammonium-nitrate medium there was no difference in main root length between WL and WL+FR (Figure 5B). To ensure that this effect was due to the ammonium addition and not to the concomitant reduction of available nitrate, we included an additional control where we added 0.67 
$\mathrm{mM}$ ammonium in addition to the $2 \mathrm{mM}$ nitrate and a medium with $1.33 \mathrm{mM}$ nitrate as only N-source (Figure S4B, Table 1). Again, we saw that low $(0.2 \mathrm{mM})$ nitrate led to a lower lateral density without a WL+FR-induced reduction and that the combined nitrate-ammonium medium had a higher lateral root density that was not affected by WL+FR. Adding $0.67 \mathrm{mM}$ ammonium to $2 \mathrm{mM}$ or to $1.33 \mathrm{mM}$ nitrate gave the same results, indicating that in the combined-N media, it is the variation in ammonium, not nitrate, that affects the phenotypes (Figure S4B). The mild depletion of nitrate (1.33 $\mathrm{mM}$ ) was comparable to $2 \mathrm{mM}$ nitrate; the lateral root density in WL was similar and WL+FR led to a reduction in lateral root density (Figure S4B).

On the combined nitrate-ammonium medium we observed a decrease in NRT2.1 expression (Figure 4D). It is known that NRT2.1 expression in ammonium-containing medium is derepressed in the NRT1.1 mutant chl1-5 (Muños et al., 2004; Bouguyon et al., 2015). Therefore, we tested the chl1-5 mutant on normal and low nitrate. chll-5 had a similar lateral root density as Col-0 in WL normal nitrate, however, in WL+FR it had a higher lateral root density, and not a lower lateral root density (Col-0) or no difference between WL and WL+FR (nrt2.1 nrt2.2) (Fig. S4B). This suggests that the response of the nrt2.1 nrt2.2 mutant on nitrate only-N media is specific to these transporters and not generic to any nitrate transporter. Interestingly the expression of NRT1.1 could also be induced by WL+FR, and future studies would be required to investigate how this would functionally integrate with the WL+FR response. Our findings on nitrate versus combined nitrate-ammonium media show the importance of the nitrogen source, as compared to the amount of available nitrogen. These findings also indicate that the WL and WL+FR effects we observe in normal and low nitrate and the differences due to ammonium are likely due to changes in signaling rather than a limiting effect of the available nitrogen for growth. Lateral root density of $n r t 2.1$ nrt2.2 on the combined nitrateammonium medium was similar between WL and WL+FR and similar to Col-0 on the same medium, indicating that the ammonium-related root architectures are independent of $n r t 2.1$ nrt2.2 and insensitive to supplemental FR.

\section{Conclusion and Discussion}

Low R:FR signaling indicates nearby vegetation and induces complex developmental outputs in shade intolerant plants. In the shoot of young seedlings the relative increase in FR light leads to increased elongation, while in the root it leads to a reduction in root elongation and lateral root 
formation (van Gelderen et al., 2018). Here we have shown that a reduction in nitrate levels reduces the WL+FR response of the shoot and the root. However, it is only the shoot that detects the FR light in the experiments presented here, because we made use of the D-root system. HY5 appears to acts as a shoot-to-root signal that links the perception of FR light in the shoot to the root possibly via shootto-root transport, or via unknown intermediates (van Gelderen et al., 2018; Chen et al., 2016). Both nrt2.1 nrt2.2 and hy5 hyh mutants lack a lateral root density response to WL+FR. However, these mutants did respond differently to WL+FR and also to low nitrate. It was striking that the hy5 hyh mutant root development was insensitive to very low nitrate levels, irrespective of the R:FR light ratio. This indicates that HY5 can play a central role in the adaptation of the root system to low nitrate, as well as to shoot-perceived WL+FR. The fact that on normal nitrate $n r t 2.1 \mathrm{nrt} 2.2 \mathrm{did}$ not show a reduction in lateral root density upon supplemental FR exposure shows that it is involved in this response when nitrate conditions are not limiting. Overall, we can conclude that it is likely that NRT2.1 has a positive effect on lateral root development, since the $n r t 2.12 .2$ mutant had a slightly

312 lower lateral root density compared to Col-0 and that HY5 has a negative effect, since the mutant had

313 a higher lateral root density. It is not surprising that the nrt2.1 nrt2.2 mutant is more sensitive to low nitrate, since it probably has an impaired nitrate uptake (Remans et al., 2006). Therefore, it is a

315 distinct possibility is that the low nitrate insensitivity of hy5 hyh (Figure S1B) is due to the increase in NRT2.1 transcript, thereby tentatively enhancing nitrate uptake, making it less sensitive to nitrate reduction in the medium. However, we cannot conclude this for the whole seedling, since low R:FRinduced hypocotyl elongation in hy5 hyh is still affected by low nitrate.

It is very interesting that low nitrate led to an increase in $H Y 5$ expression. HY5 overexpressing lines have a reduced lateral root density and do not have a reduced lateral root growth due to shoot perceived WL+FR (Sibout et al., 2006; van Gelderen et al., 2018). Our qPCR data are consistent with the suggestion that NRT2.1 acts downstream of HY5 in the lateral root density WL+FR response. Furthermore, NRT2.1 was upregulated in the root when the shoot was in WL+FR and this upregulation was dependent upon HY5. This is in accordance with our finding that NRT2.1 is important for the response to WL+FR. Thereby we suggest a model (Figure 6) where low R:FR induces and stabilizes HY5 in the shoot, after which it could be transported to the root, where through a positive feedback mechanism it stimulates its own transcription (Zhang et al., 2017). This

328 leads to repression of lateral root development, directly via for example ARF19 (van Gelderen et al., 2018), and indirectly via repression of NRT2.1. In low nitrate conditions, $N R T 2.1$ is upregulated at

330 first, but this effect is transient (Okamoto et al., 2003), which explains the relatively mild induction 


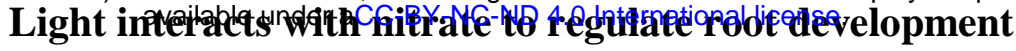

331 of NRT2.1 observed in plants exposed to low nitrate for $5 \mathrm{~d}$ (Figure 4C). However, low nitrate also

332 stimulates HY5 expression (Fig. 4F), which would likely reduce lateral root density (Sibout et al.,

333 2006; van Gelderen et al., 2018).

334 From the mutant analysis we conclude that NRT2.1 has a positive effect on root growth. The nutrient

335 context is crucial for the effect of the nrt2.1 mutation (Little et al., 2005; Remans et al., 2006). We

336 found this as well, since the positive effect of NRT2.1 on lateral root formation was only true for

337 nitrate-only-N media, as the nrt2.1 nrt2.2 mutant had a wild type lateral root density and main root

338 length when ammonium was used in addition to nitrate. The simplest explanation for this result is

339 that ammonium directly stimulates lateral root outgrowth via acidification of the apoplast, leading to

340 increased pH-dependent auxin transport (Meier et al., 2020). This could also mask the strong

341 inhibitory effect of ammonium on NRT2.1 transcription (Muños et al., 2004; Bouguyon et al., 2015),

342 since negative effects of reduced NRT2.1 on lateral root development would be counteracted by $\mathrm{pH}-$

343 driven auxin transport. Increased NRT2.1 expression in the hy5 hyh mutant is opposite of the result

344 obtained by Chen et al., 2016, but this is only true in nitrate-only-N media. When tested on medium

345 with supplemented ammonium, or $1 / 2$ MS medium, our data were consistent with Chen et al., 2016,

346 who also used MS medium. These expression results with combined nitrate-ammonium media do

347 highlight that the effect of HY5 on the transcription of downstream genes is not always black and

348 white and also relies upon other factors (Burko et al., 2020).

349 In this study we tried to answer the question how a plant can integrate different signals coming from

350 the shoot and the root. When a plant is competing for available light it is important to adjust its

351 development. However, it is possible that it will only do so when it can afford to. In other words,

352 only when there are enough nutrients will the plant sacrifice some development of the root system.

353 These carbohydrates are very useful for investing in short-term shoot growth. However, we show

354 here that under nutrient-depleted conditions, root system development does not respond to shoot-

355 detected FR anymore, probably safe-guarding nutrient uptake possibilities.

356 Concluding, we have shown that nitrate levels can modulate the response to low R:FR-induced

357 stimuli of neighbour competition and that this integration involves the HY5 transcription factor and

358 that the NRT2.1 nitrate transporter play an important role in this integration. It is not yet clear exactly

359 how NRT2.1 affects lateral root development. It could be that it is due to its ability as a nitrate

360 transporter, but possibly also as an active signaling component. Since NRT2.1 acts as a

361 transporter/receptor, it has also been put forward that NRT2.1 could affect lateral root outgrowth via 


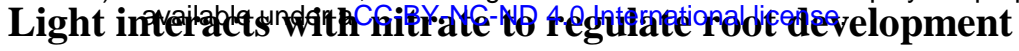

362 differential expression of aquaporins ( $\mathrm{Li}$ et al., 2016). In this way, NRT2.1 could locally stimulate

363 water uptake and turgor pressure of the cells around the lateral root primordium, affecting lateral root

364 emergence. Elucidating the exact mechanisms through which NRT2.1 regulates lateral root

365 development responses to nitrate and light are important questions for future research.

366

3675 Tables

$368 \quad 5.1 \quad$ Table 1: Non-1/2MS-nutrient media compositions

\begin{tabular}{|c|c|c|c|c|c|c|c|}
\hline macronutrients & $\begin{array}{c}\text { normal } \mathrm{N} \\
\text { (2) } \mathrm{mM}\end{array}$ & $\begin{array}{c}\text { low } N \\
(0.2) \mathrm{mM}\end{array}$ & $\begin{array}{c}\text { lower N } \\
(0.05) \mathrm{mM}\end{array}$ & $\begin{array}{c}\mathrm{NH} 4+\mathrm{NO} 3 \\
\text { (2) } \mathrm{mM}\end{array}$ & $\begin{array}{c}1.33 \mathrm{NO3} \\
(1.33) \mathrm{mM}\end{array}$ & $\begin{array}{c}+\mathrm{NH} 4 \\
(2.67) \mathrm{mM}\end{array}$ & $\begin{array}{c}\text { low } P \\
(2 / 0.02) \mathrm{ml}\end{array}$ \\
\hline Potassium nitrate $\left(\mathrm{KNO}_{3}\right)$ & 2.00 & 0.20 & 0.05 & 0.67 & 1.33 & 2.00 & 2.0 \\
\hline Ammonium nitrate $\left(\mathrm{NH}_{4} \mathrm{NO}_{3}\right)$ & & & & 0.67 & & 0.67 & \\
\hline potassium chloride $(\mathrm{KCl})$ & & 1.80 & 1.95 & 1.33 & 0.67 & 1.95 & \\
\hline Calcium chloride $\left(\mathrm{CaCl}_{2} \cdot 2 \mathrm{H}_{2} \mathrm{O}\right)$ & 0.25 & 0.25 & 0.25 & 0.25 & 0.25 & 0.25 & 0.2 \\
\hline Magnesium chloride $\left(\mathrm{MgCL}_{2} \cdot 6 \mathrm{H}_{2} \mathrm{O}\right)$ & 0.25 & 0.25 & 0.25 & 0.25 & 0.25 & 0.25 & 0.2 \\
\hline Magnesium sulphate $\left(\mathrm{MgSO}_{4} \cdot 7 \mathrm{H}_{2} \mathrm{O}\right)$ & 0.25 & 0.25 & 0.25 & 0.25 & 0.25 & 0.25 & 0.2 \\
\hline sodium phosphate $\left(\mathrm{NaH}_{2} \mathrm{PO}_{4}\right)$ & 0.50 & 0.50 & 0.50 & 0.50 & 0.50 & 0.50 & 0.0 \\
\hline sodium chloride $(\mathrm{NaCl})$ & 8.00 & 8.00 & 8.00 & 7.33 & 8.00 & 8.00 & 8.4 \\
\hline
\end{tabular}

\begin{tabular}{|c|c|c|c|c|c|c|c|}
\hline micronutrients & $\begin{array}{c}\text { normal N } \\
\text { (2) } \mathrm{mM}\end{array}$ & $\begin{array}{c}\text { low } \mathrm{N} \\
(0.2) \mathrm{mM}\end{array}$ & $\begin{array}{c}\text { lower N } \\
(0.05) \mathrm{mM}\end{array}$ & $\begin{array}{c}\mathrm{NH} 4+\mathrm{NH} 3 \\
\text { (2) } \mathrm{mM}\end{array}$ & $\begin{array}{c}1.33 \mathrm{NO3} \\
(1.33) \mathrm{mM}\end{array}$ & $\begin{array}{c}+\mathrm{NH} 4 \\
(2.67) \mathrm{mM}\end{array}$ & $\begin{array}{c}\text { low } P \\
(2 / 0.02) \mathrm{ml}\end{array}$ \\
\hline $\mathrm{Fe}(\mathrm{III}) \mathrm{Na}-\mathrm{EDTA}$ & 0.04 & 0.04 & 0.04 & 0.04 & 0.04 & 0.04 & 0.0 \\
\hline $\mathrm{MnCl}_{2} \cdot 4 \mathrm{H}_{2} \mathrm{O}$ & 1.80 & 1.80 & 1.80 & 1.80 & 1.80 & 1.80 & 1.8 \\
\hline $\mathrm{H}_{3} \mathrm{BO}_{3}$ & 45.00 & 45.00 & 45.00 & 45.00 & 45.00 & 45.00 & 45.0 \\
\hline $\mathrm{ZnSO}_{4} 7 \mathrm{H}_{2} \mathrm{O}$ & 0.38 & 0.38 & 0.38 & 0.38 & 0.38 & 0.38 & 0.3 \\
\hline$\left(\mathrm{NH}_{4}\right)_{6} \mathrm{Mo}_{7} \mathrm{O}_{24}$ & 0.02 & 0.02 & 0.02 & 0.02 & 0.02 & 0.02 & 0.0 \\
\hline $\mathrm{CuSO}_{4} \cdot 5 \mathrm{H}_{2} \mathrm{O}$ & 0.16 & 0.16 & 0.16 & 0.16 & 0.16 & 0.16 & 0.1 \\
\hline $\mathrm{CoCl}_{2}$ & 0.01 & 0.01 & 0.01 & 0.01 & 0.01 & 0.01 & 0.0 \\
\hline
\end{tabular}

\begin{tabular}{|c|c|c|c|c|c|c|c|}
\hline Vitamins and organics & $\begin{array}{c}\text { normal N } \\
\text { (2) } \mathrm{mM}\end{array}$ & $\begin{array}{c}\text { low N } \\
(0.2) \mathrm{mM}\end{array}$ & $\begin{array}{c}\text { lower N } \\
(0.05) \mathrm{mM}\end{array}$ & $\begin{array}{c}\mathrm{NH} 4+\mathrm{NH} 3 \\
\text { (2) } \mathrm{mM}\end{array}$ & $\begin{array}{r}1.33 \mathrm{NO3} \\
(1.33) \mathrm{mM}\end{array}$ & $\begin{array}{c}+\mathrm{NH} 4 \\
(2.67) \mathrm{mM}\end{array}$ & $\begin{array}{c}\text { low } P \\
(2 / 0.02) \mathrm{ml}\end{array}$ \\
\hline myo-Inositol 100 mg/l & 0.2775 & 0.2775 & 0.2775 & 0.2775 & 0.2775 & 0.2775 & 0.277 \\
\hline Niacin $0.5 \mathrm{mg} / \mathrm{l}$ & 0.0020 & 0.0020 & 0.0020 & 0.0020 & 0.0020 & 0.0020 & 0.002 \\
\hline Pyridoxine $\cdot \mathrm{HCl} 0.5 \mathrm{mg} / \mathrm{l}$ & 0.0012 & 0.0012 & 0.0012 & 0.0012 & 0.0012 & 0.0012 & 0.001 \\
\hline Thiamine $\cdot \mathrm{HCl} 0.1 \mathrm{mg} / \mathrm{l}$ & 0.0001 & 0.0001 & 0.0001 & 0.0001 & 0.0001 & 0.0001 & 0.000 \\
\hline Glycine (recrystallized) $2.0 \mathrm{mg} / \mathrm{l}$ & 0.0133 & 0.0133 & 0.0133 & 0.0133 & 0.0133 & 0.0133 & 0.013 \\
\hline
\end{tabular}

369

$370 \quad 5.2 \quad$ Table 2: Primers used in study. 


\begin{tabular}{|c|c|c|}
\hline Primer name & sequence & Use \\
\hline APT1 FW & AATGGCGACTGAAGATGTGC & qPCR housekeeping gene \\
\hline APT1 REV & TCAGTGTCGAGAAGAAGCGT & qPCR housekeeping gene \\
\hline AT1G13320_fwd & GTAGGACCGGAGCCAACTAG & qPCR housekeeping gene \\
\hline AT1G13320_rev & ACAGGGAAGAATGTGCTGGA & qPCR housekeeping gene \\
\hline NRT2.1 Fw & TGGAGAAAGCGGGAGAAGTT & qPCR NRT2.1 \\
\hline NRT2.1 Rev & GCTCAACTCAACTCCCATGG & qPCR NRT2.1 \\
\hline NRT2.2 Fw & CGCCGTTACAAATTACAGGAC & qPCR NRT2.2 \\
\hline NRT2.2 Rev & ATAATCCCCGCTGTGTGAAG & qPCR NRT2.2 \\
\hline HY5 qPCR Fw & TGAGCGAGTTGGAAAACAGA & qPCR HY5 \\
\hline HY5 qPCR Rev & AAGGCTTGCATCAGCATTAG & qPCR HY5 \\
\hline NRT1.1 qPCRfw & TATTATTGCGGCGGAAGG & qPCR NRT1.1 \\
\hline NRT1.1 qPCRrev & CGCTTCCTGATCCCTTATTG & qPCR NRT1.1 \\
\hline chl1-5 p1 & CCCGAGAGCTCAGTTTGAGA & chl1-5 genotyping \\
\hline chl1-5 p2 & TATCCTTCACACACATGCATGAC & chl1-5 genotyping \\
\hline chl1-5 p2 & TGGGGTGATCCGTAAGATTC & chl1-5 genotyping \\
\hline SALK_035429 LP & TGATCCAAAGTTCCCTCCAG & nrt2.1 nrt2.2 genotyping \\
\hline SALK_035429 RP & GTTGCCTCTGTCTCTGGGAG & nrt2.1 nrt2.2 genotyping \\
\hline
\end{tabular}

\section{Conflict of Interest}

373 The authors declare that the research was conducted in the absence of any commercial or financial

374 relationships that could be construed as a potential conflict of interest.

\section{$375 \quad 7 \quad$ Author Contributions}


K.v.G. and R.P. designed the experiments and wrote the manuscript. K.v.G., C.K. and P.L. performed and analysed experiments.

\section{$\begin{array}{lll}378 & 8 & \text { Funding }\end{array}$}

379 This research was funded by the Netherlands Organisation for Scientific Research, open competition 380 grant 823.01.013 and Vici grant 865.17.002 to R.P., a scholarship of government sponsorship for overseas study, Taiwan, admission number 0991167-2-UK-004 to C.K.

\section{Acknowledgments}

The authors would like to thank Dr. Sandrine Ruffel for providing the chll-5 mutant and for suggestions on the direction of the project. Special thanks go out to Jannetje Kooij and Koen Bensink for practical help during the project.

\section{References}

Ballaré, C.L. and Pierik, R. (2017). The shade-avoidance syndrome: Multiple signals and ecological consequences. Plant Cell Environ. 40: 2530-2543.

Bouguyon, E. et al. (2015). Multiple mechanisms of nitrate sensing by Arabidopsis nitrate transceptor NRT1.1. Nat. Plants 1: 15015.

Bouguyon, E., Perrine-Walker, F., Pervent, M., Rochette, J., Cuesta, C., Benkova, E., Martinière, A., Bach, L., Krouk, G., Gojon, A., and Nacry, P. (2016). Nitrate Controls Root Development through Post-Transcriptional Regulation of the NRT1.1/NPF6.3

393

394 transporter/sensor. Plant Physiol. 172: pp.01047.2016.

Burko, Y., Seluzicki, A., Zander, M., Pedmale, U. V, Ecker, J.R., and Chory, J. (2020). Chimeric Activators and Repressors Define HY5 Activity and Reveal a Light-Regulated Feedback Mechanism. Plant Cell 32: 967 LP-983.

Cerezo, M., Tillard, P., Filleur, S., Muños, S., Daniel-Vedele, F., and Gojon, A. (2001). Major Alterations of the Regulation of Root NO\&lt;sub\&gt;3\&lt;/sub\&gt; \&lt;sup\&gt;-\&lt;/sup\&gt; Uptake Are Associated with the Mutation of \&lt;em\&gt;Nrt2.1\&lt;/em\&gt; and \&lt;em\&gt;Nrt2.2\&lt;/em\&gt; Genes in Arabidopsis. Plant Physiol. 127: 262 LP-271.

Chen, M. and Chory, J. (2011). Phytochrome signaling mechanisms and the control of plant development. Trends Cell Biol. 21: 664-671.

Chen, X., Yao, Q., Gao, X., Jiang, C., Harberd, N.P., and Fu, X. (2016). Shoot-to-Root Mobile Transcription Factor HY5 Coordinates Plant Carbon and Nitrogen Acquisition. Curr. Biol. 26: 640-646.

Cluis, C.P., Mouchel, C.F., and Hardtke, C.S. (2004). The Arabidopsis transcription factor HY5 integrates light and hormone signaling pathways. Plant J. 38: 332-47.

van Gelderen, K., Kang, C., Paalman, R., Keuskamp, D., Hayes, S., and Pierik, R. (2018). Farred light detection in the shoot regulates lateral root development through the HY5 transcription factor. Plant Cell 30: 101-116. 
Van Gelderen, K., Kang, C., and Pierik, R. (2018). Light signaling, root development, and plasticity. Plant Physiol. 176: 1049-1060.

Gruber, B.D., Giehl, R.F.H., Friedel, S., and von Wirén, N. (2013). Plasticity of the Arabidopsis root system under nutrient deficiencies. Plant Physiol. 163: 161-79.

Hachiya, T. and Sakakibara, H. (2017). Interactions between nitrate and ammonium in their uptake, allocation, assimilation, and signaling in plants. J. Exp. Bot. 68: 2501-2512.

Jonassen, E.M., Lea, U.S., and Lillo, C. (2008). HY5 and HYH are positive regulators of nitrate

Jonassen, E.M., Sandsmark, B.A.A., and Lillo, C. (2009). Unique status of NIA2 in nitrate assimilation: NIA2 expression is promoted by $\mathrm{HY} 5 / \mathrm{HYH}$ and inhibited by PIF4. Plant Signal. Behav. 4: 1084-1086.

423

424

Kellermeier, F., Armengaud, P., Seditas, T.J., Danku, J., Salt, D.E., and Amtmann, A. (2014). Analysis of the Root System Architecture of Arabidopsis Provides a Quantitative Readout of Crosstalk between Nutritional Signals. Plant Cell 26: 1480-1496.

Krouk, G., Tillard, P., and Gojon, A. (2006). Regulation of the high-affinity NO3- uptake system by NRT1.1-mediated NO3- demand signaling in Arabidopsis. Plant Physiol. 142: 1075-1086.

Leivar, P. and Monte, E. (2014). PIFs: Systems Integrators in Plant Development. Plant Cell 26: $56-78$.

Li, G., Tillard, P., Gojon, A., and Maurel, C. (2016). Dual regulation of root hydraulic conductivity and plasma membrane aquaporins by plant nitrate accumulation and high-affinity nitrate transporter NRT2.1. Plant Cell Physiol. 57: 733-742.

Li, W., Wang, Y., Okamoto, M., Crawford, N.M., Siddiqi, M.Y., and Glass, A.D.M. (2007). Dissection of the AtNRT2.1:AtNRT2.2 inducible high-affinity nitrate transporter gene cluster. Plant Physiol. 143: 425-433.

Lima, J.E., Kojima, S., Takahashi, H., and von Wirén, N. (2010). Ammonium triggers lateral root branching in Arabidopsis in an AMMONIUM TRANSPORTER1;3-dependent manner. Plant Cell 22: 3621-33.

Little, D.Y., Rao, H., Oliva, S., Daniel-Vedele, F.F., Krapp, A., and Malamy, J.E. (2005). The putative high-affinity nitrate transporter NRT2.1 represses lateral root initiation in response to nutritional cues. Proc. Natl. Acad. Sci. U. S. A. 102: 13693-13698. 
Meier, M., Liu, Y., Lay-Pruitt, K.S., Takahashi, H., and von Wirén, N. (2020). Auxin-mediated root branching is determined by the form of available nitrogen. Nat. Plants 6: 1136-1145.

Mounier, E., Pervent, M., Ljung, K., Gojon, A., and Nacry, P. (2014). Auxin-mediated nitrate signalling by NRT1.1 participates in the adaptive response of Arabidopsis root architecture to the spatial heterogeneity of nitrate availability. Plant, Cell Environ. 37: 162-174.

Muños, S., Cazettes, C., Fizames, C., Gaymard, F., Tillard, P., Lepetit, M., Lejay, L., and Gojon, A. (2004). Transcript Profiling in the \&lt;em\&gt;chl1-5\&lt;/em\&gt; Mutant of Arabidopsis Reveals a Role of the Nitrate Transporter NRT1.1 in the Regulation of Another Nitrate Transporter, NRT2.1. Plant Cell 16: 2433 LP-2447.

O’Brien, J.A.A., Vega, A., Bouguyon, E., Krouk, G., Gojon, A., Coruzzi, G., and Gutiérrez, R.A.A. (2016). Nitrate Transport, Sensing, and Responses in Plants. Mol. Plant 9: 837-856.

Okamoto, M., Vidmar, J.J., and Glass, A.D.M. (2003). Regulation of NRT1 and NRT2 gene families of Arabidopsis thaliana: Responses to nitrate provision. Plant Cell Physiol. 44: 304317.

Oyama, T., Shimura, Y., and Okada, K. (1997). The Arabidopsis HY5 gene encodes a bZIP protein that regulates stimulus- induced development of root and hypocotyl. Genes Dev. 11: 2983-2995.

Sakuraba, Y., Kanno, S., Mabuchi, A., Monda, K., Iba, K., and Yanagisawa, S. (2018). A phytochrome B-mediated regulatory mechanism of phosphorus acquisition. Nat. Plants 4.

Salisbury, F.J., Hall, A., Grierson, C.S., Halliday, K.J., Buildings, K., Road, M., Building, B., and Street, C. (2007). Phytochrome coordinates Arabidopsis shoot and root development. Plant J. 50: 429-38.

Sibout, R., Sukumar, P., Hettiarachchi, C., Holm, M., Muday, G.K., and Hardtke, C.S. (2006). Opposite root growth phenotypes of hy5 versus hy5 hyh mutants correlate with increased constitutive auxin signaling. PLoS Genet. 2: e202.

Silva-Navas, J. et al. (2015). D-Root: A system for cultivating plants with the roots in darkness or under different light conditions. Plant J. 84: 244-255.

Zhang, Y., Li, C., Zhang, J., Wang, J., Yang, J., Lv, Y., Yang, N., Liu, J., Wang, X., Palfalvi, G., Wang, G., and Zheng, L. (2017). Dissection of HY5/HYH expression in Arabidopsis reveals a root-autonomous HY5-mediated photomorphogenic pathway. PLoS One 12: 1-15. 


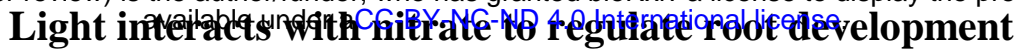
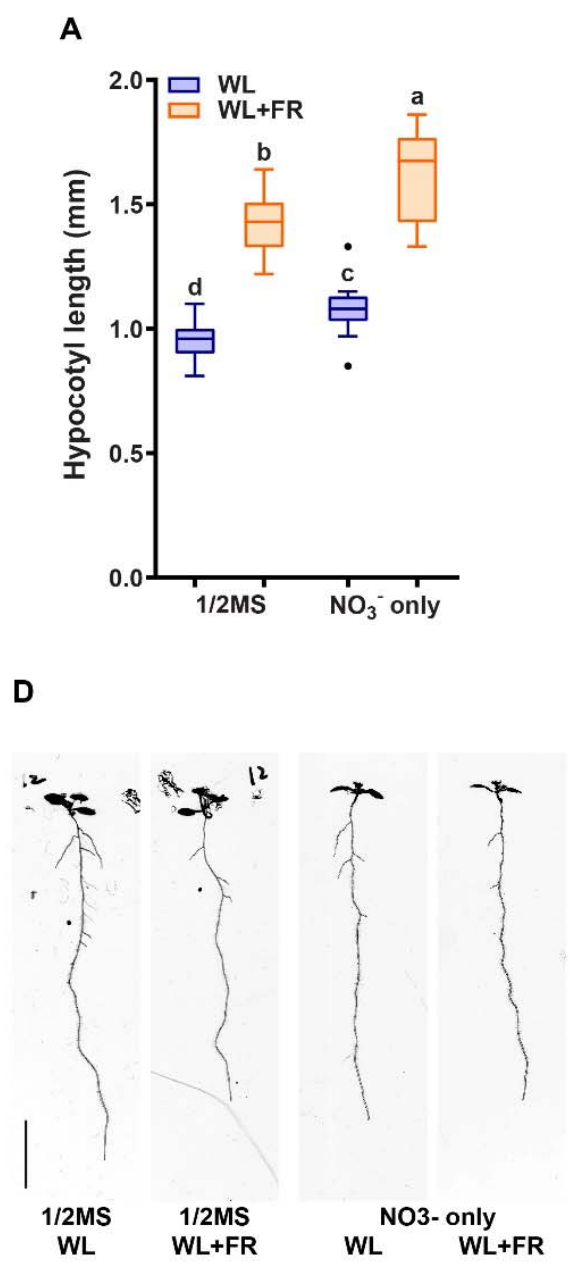

G

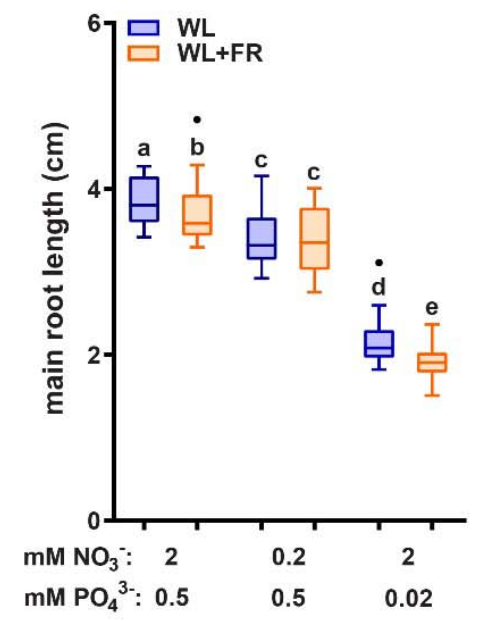

B

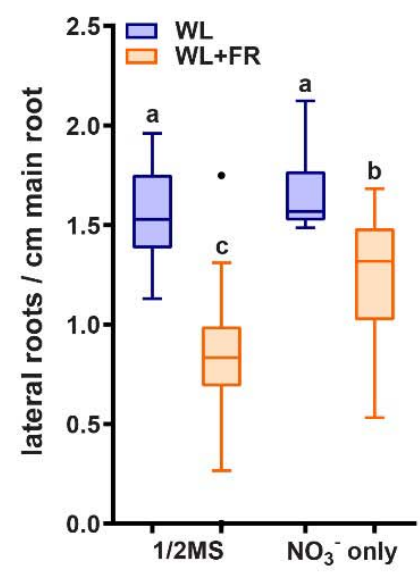

E

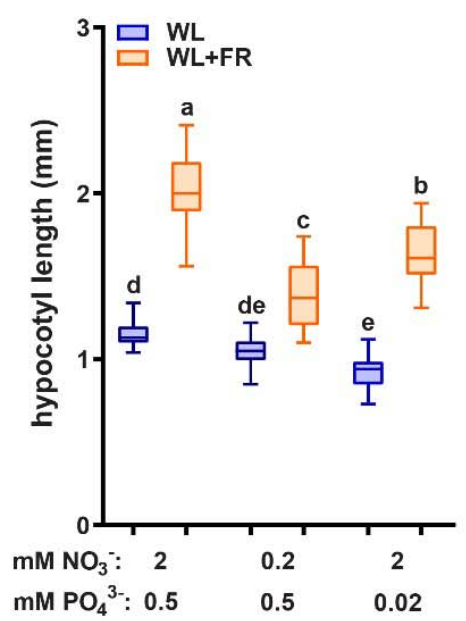

C

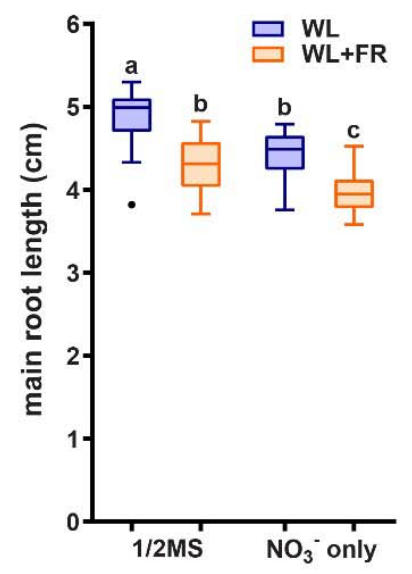

F

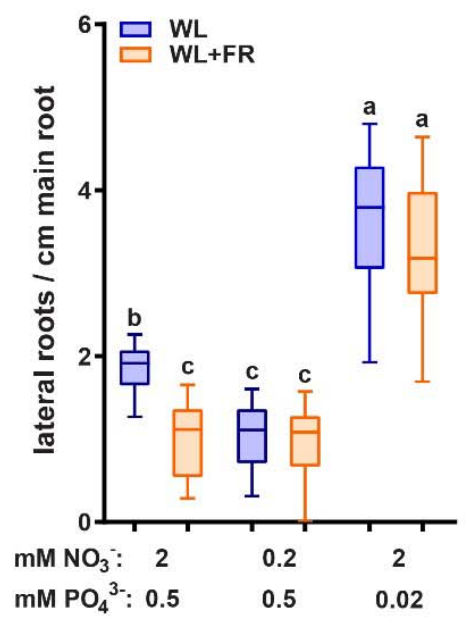

H

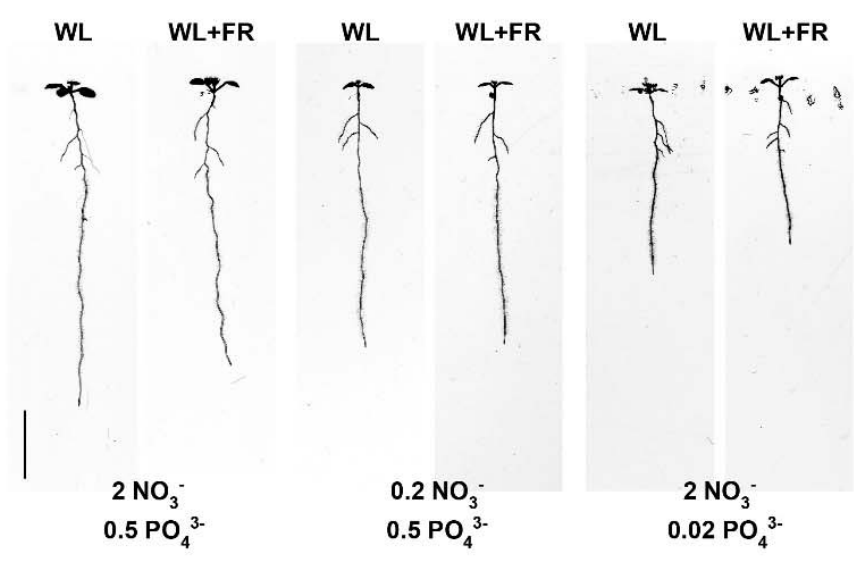

Figure 1: Nutrient shortage decreases the response to low R:FR in both the hypocotyl and root.

485 (A-D) Seedlings (Col-0) were grown in D-root plates (Silva-Navas et al., 2015), with WL and WL+FR light 


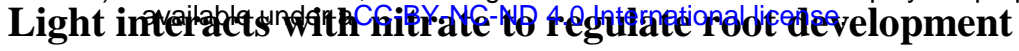

487

488

489

490

491

492

medium of (Kellermeier et al., 2014), which does not contain ammonium as a nitrogen source. (A) Hypocotyl length, (B) Main root length, (C) Lateral root density. (D) Representative seedlings for data in A,B and C. (E$\mathrm{H})$ Analysis of 8-day-old seedlings grown in either control (2mM NO3-), low nitrate medium $(0.2 \mathrm{mM})$, or low phosphate (0.2 mM). (E) Hypocotyl length, (F) Main root length, (G) Lateral root density. (H) Representative seedlings for data in A,B and C. Letters denote statistically significant groups based on a mixed model 2-way ANOVA with posthoc tukey test $(\mathrm{p}<0.05)$. Scale bar $=1 \mathrm{~cm}$.

A

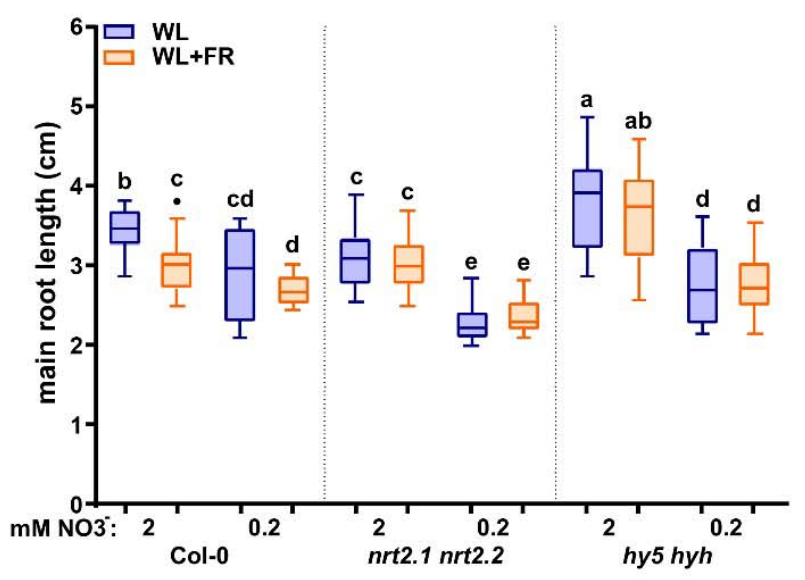

C

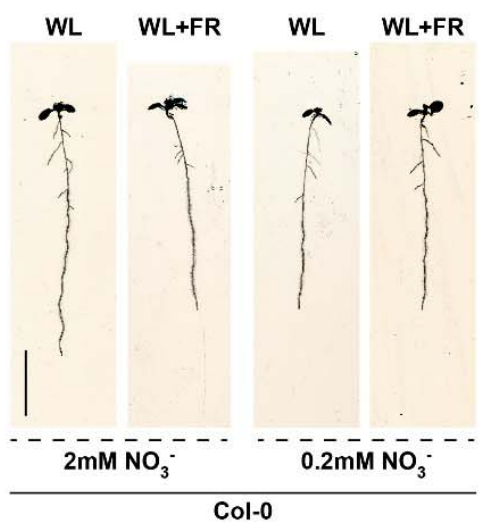

D

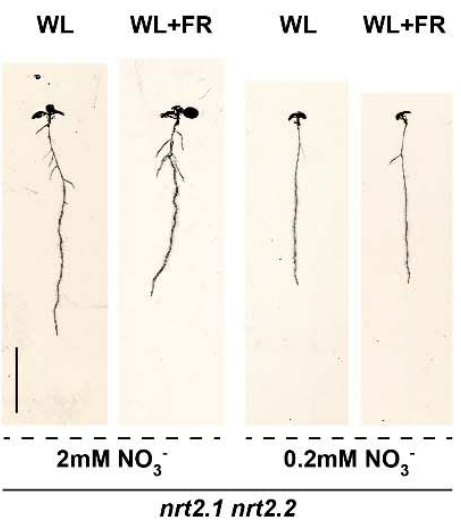

B

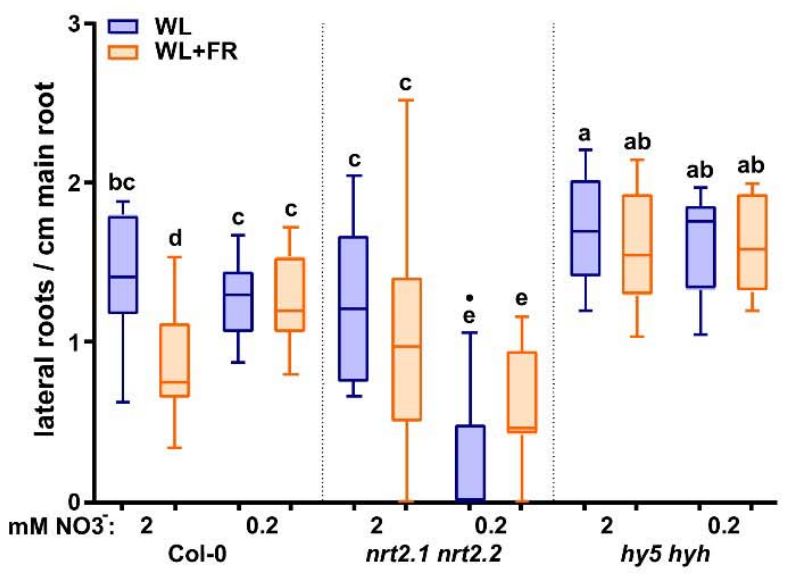

E

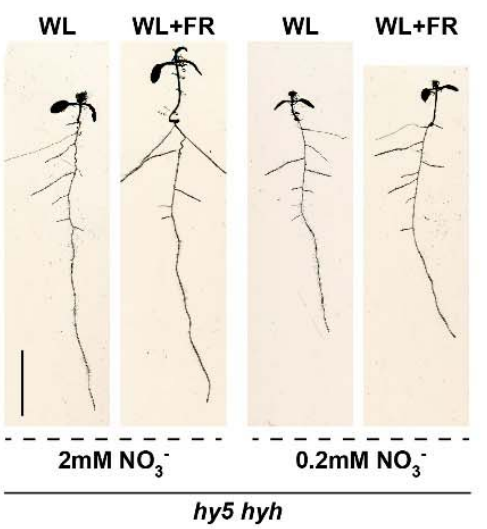

493

494

495

496

497

498
Figure 2: NRT2.1/2.2 and $H Y 5 H Y H$ are required for the combined low R:FR \& nitrate

response. Wild type Col-0, hyh5 hyh and nrt2.1 nrt2.2 seedlings grown for eight days in either WL or WL+FR on normal or low nitrate media. (A) Main root length, (B) Lateral root density. (C,D,E) Representative seedlings for data in A and B. Letters denote statistically significant groups based on a mixed model 2-way ANOVA with posthoc tukey test $(\mathrm{p}<0.05)$. Scale bar $=1 \mathrm{~cm}$. 


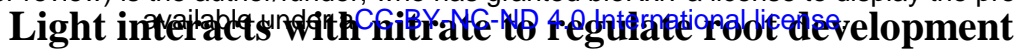

A

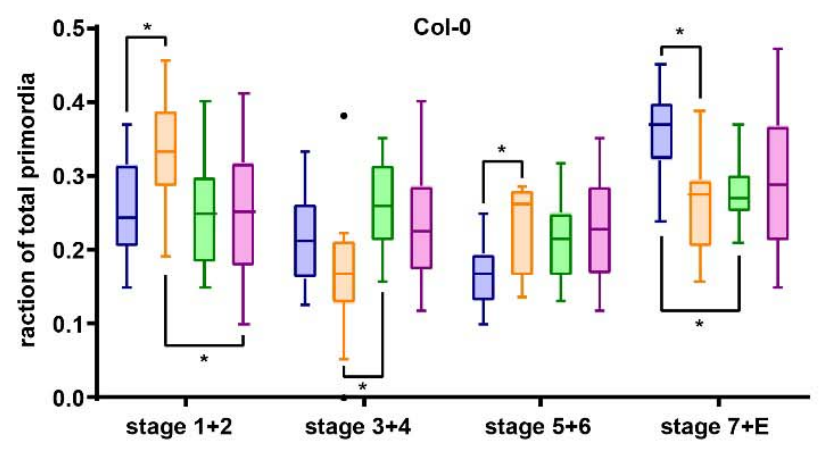

C

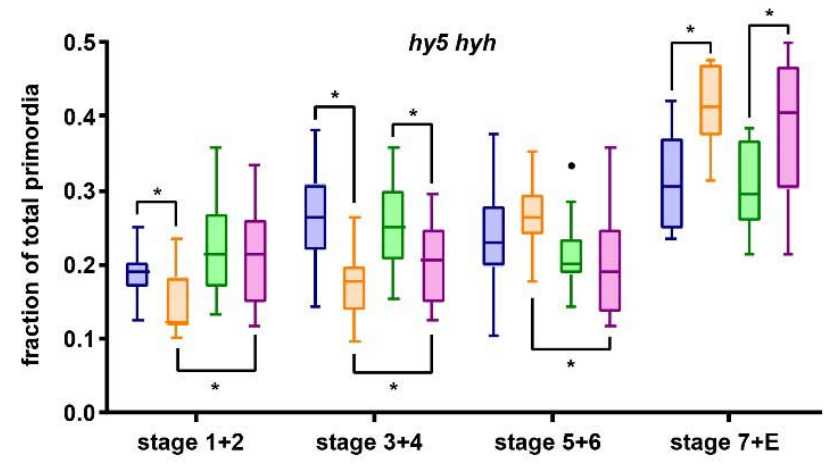

B

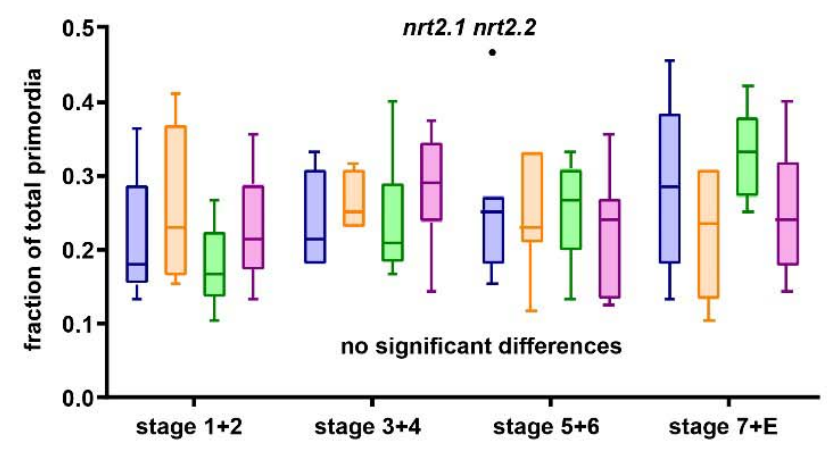

$2 \mathrm{mM} \mathrm{NO}_{3}{ }^{-} \mathrm{WL}$

$2 \mathrm{mM} \mathrm{NO}_{3}{ }^{-} \mathrm{WL}+\mathrm{FR}$

$0.2 \mathrm{mM} \mathrm{NO}_{3}^{-} \mathrm{WL}$

$0.2 \mathrm{mM} \mathrm{NO}_{3}^{-} \mathrm{WL}+\mathrm{FR}$

500 Figure 3: Low nitrate conditions regulate lateral root primordia development. Lateral root

501 primordia (LRP) data from 8-day old seedlings from experiment in Figure 2. Stages are counted

502 according to(Malamy and Benfey, 1997) and grouped in pairs of two going from early (1+2) to late

503 (7+Emerged) development. (A) Col-0, (B) nrt2.1 2.2, (C) hy5 hyh. Statistics: mixed model 2-way

504 ANOVA with post hoc Tukey test within the stage groups $(* \mathrm{p}<0.05)$. 
A

NRT2.1 expression

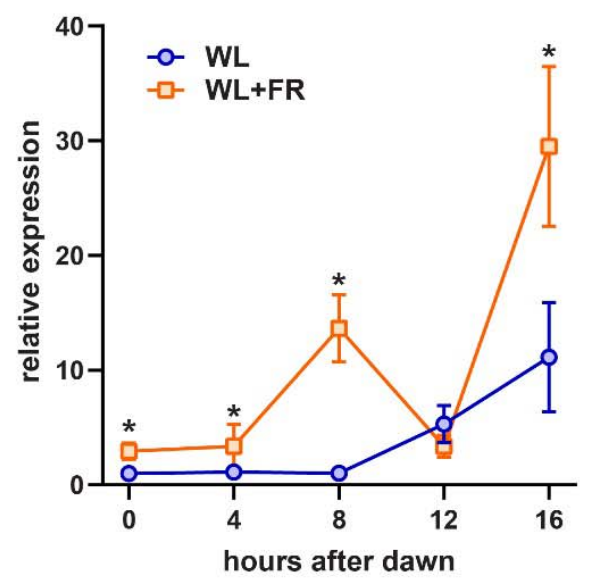

C

NRT2.1 expression

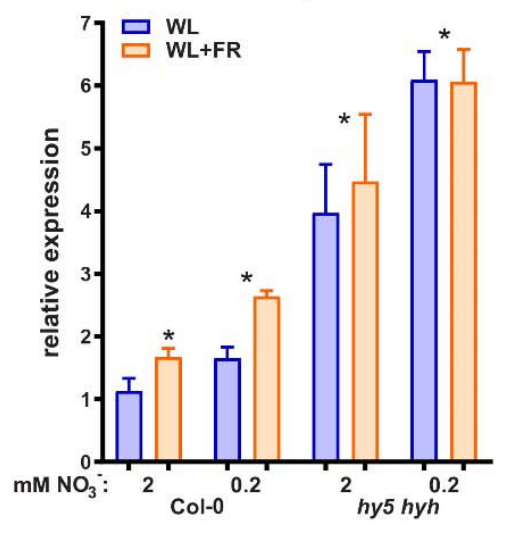

E

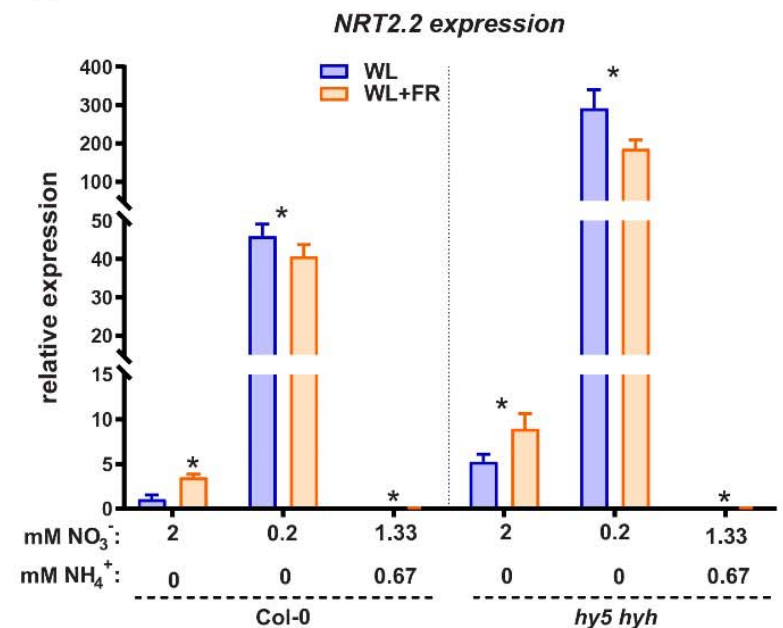

B

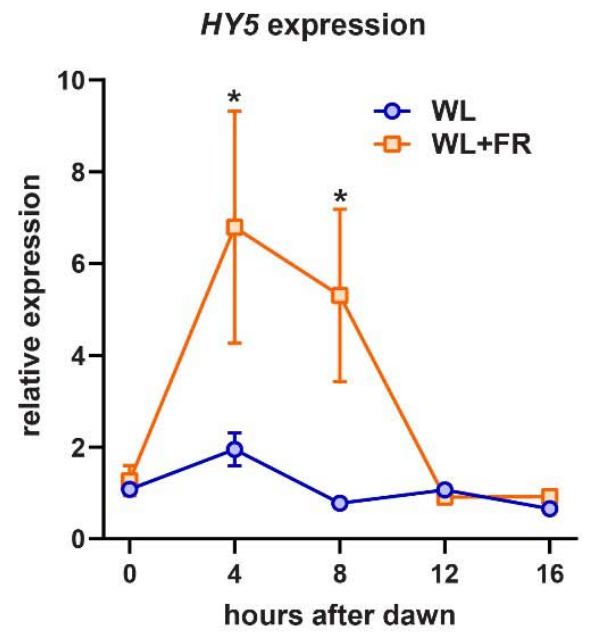

D
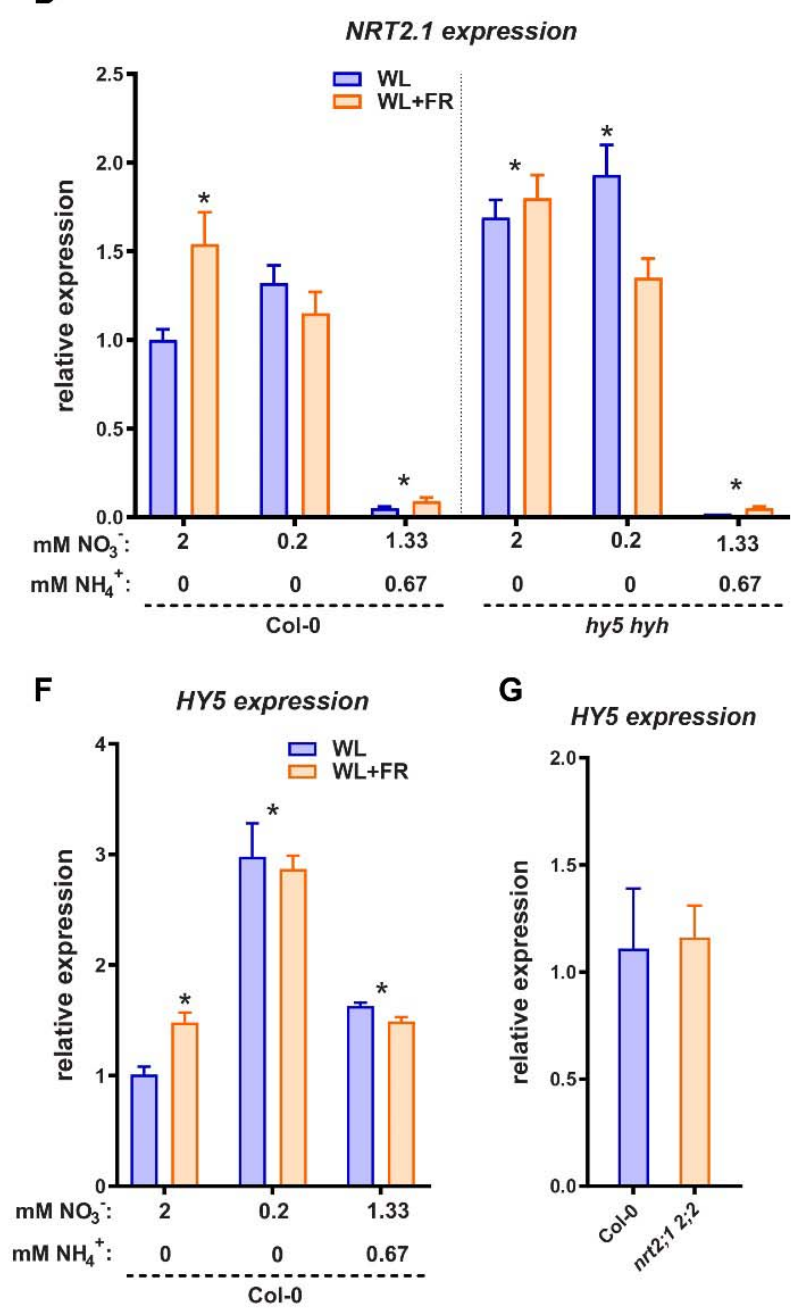

G

HY5 expression

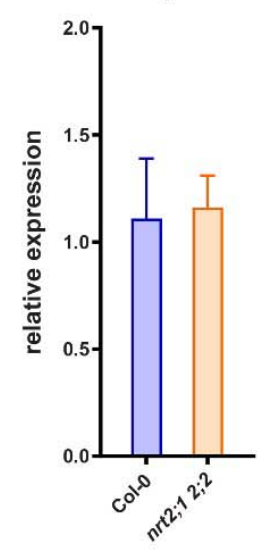

Fig. 4 HY5 and WL+FR regulate NRT2.1 expression.

508 (A,B) Time-course qPCR experiment using 5-day old seedling root material harvested between 0 50916 hours post-dawn, grown either in WL or WL+FR. WL+FR increases NRT2.1 2.2 expression in all 
510 timepoints bar 12hrs PD. HY5 expression is increased by WL+FR at 4 and 8 hours PD. (C) qPCR

511 expression analysis of NRT2.1 using RNA of 5-day old seedling roots, with combined WL/WL+FR

512 and low $\mathrm{N} /$ normal $\mathrm{N}$ treatments. (D,E,F) qPCR expression analysis similar to $(\mathrm{C})$, but with the

513 addition of a mixed nitrate/ammonium medium $\left(\mathrm{NO}_{3}{ }^{-} \mathrm{NH}_{4}{ }^{+}\right),(\mathrm{D}):$ NRT2.1 (E): NRT2.2 (F): HY5.

514 HY5 expression was not detectable in the hy5 hyh mutant. (G) qPCR expression analysis similar to

515 (C), of HY5 on normal ( $2 \mathrm{mM})$ nitrate medium in Col-0 and $n r t 2.12 .2$. (A,B) *p<0.05 WL vs

516 WL+FR with a two-way ANOVA plus post-hoc tukey test, (C-F) *p<0.05 vs 2mM Col-0 WL with a

517 one-way ANOVA plus post-hoc tukey test.

A

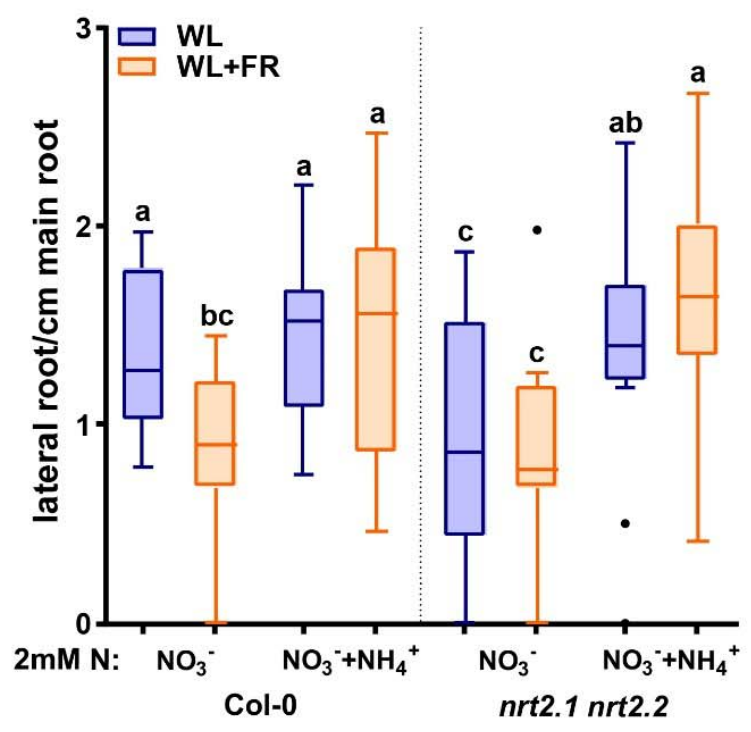

B

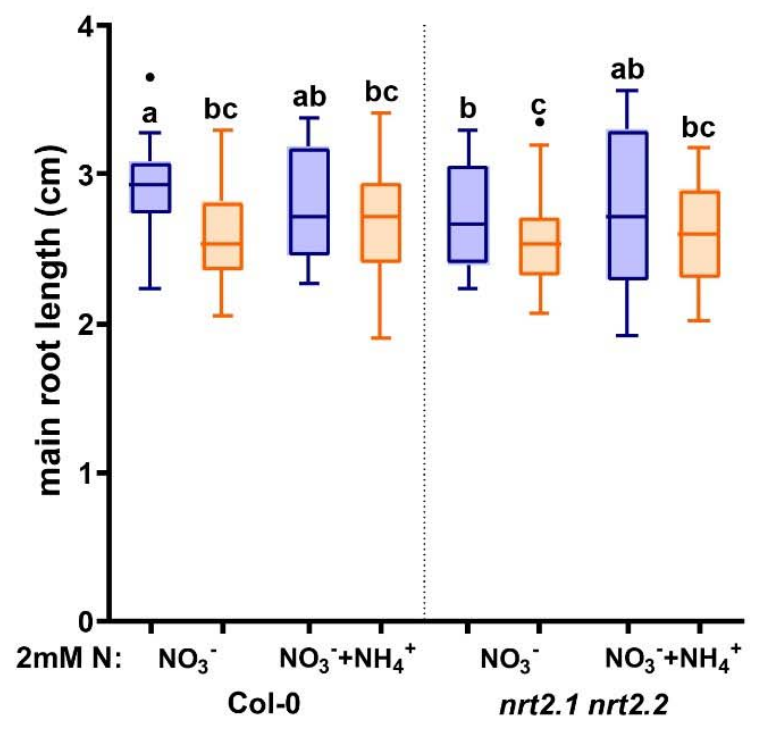

C

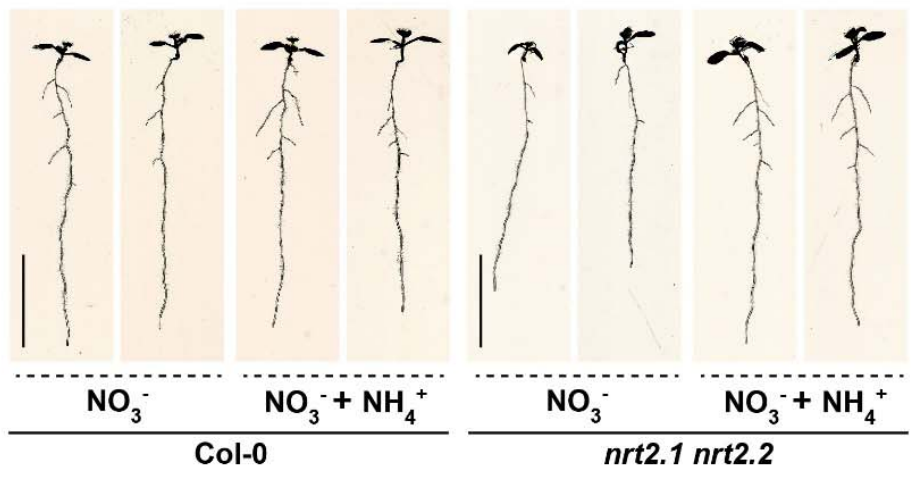

520 Figure 5: Replacing 1/3 of nitrate with ammonium can remove effect of WL+FR on root

521 development and bypass the $n r t 2.12 .2$ root phenotype. Seedlings of Col-0 and $n r t 2.1$ nrt 2.2 were 


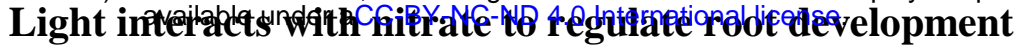

522 grown on combined nitrate-ammonium consisting of $1.33 \mathrm{mM} \mathrm{NO}_{3}{ }^{-}$, and $0.67 \mathrm{NH}_{4}{ }^{+}$. The rest of the

523 experiment was performed according to Figures $1 \& 2$. (A) hypocotyl length, (B) main root length,

524 (C) lateral root density and (D) representative 8d. old seedlings. Letters denote statistically

525 significant groups based on a mixed model 2-way ANOVA with posthoc tukey test $(\mathrm{p}<0.05)$. Scale 526 bar $=1 \mathrm{~cm}$.

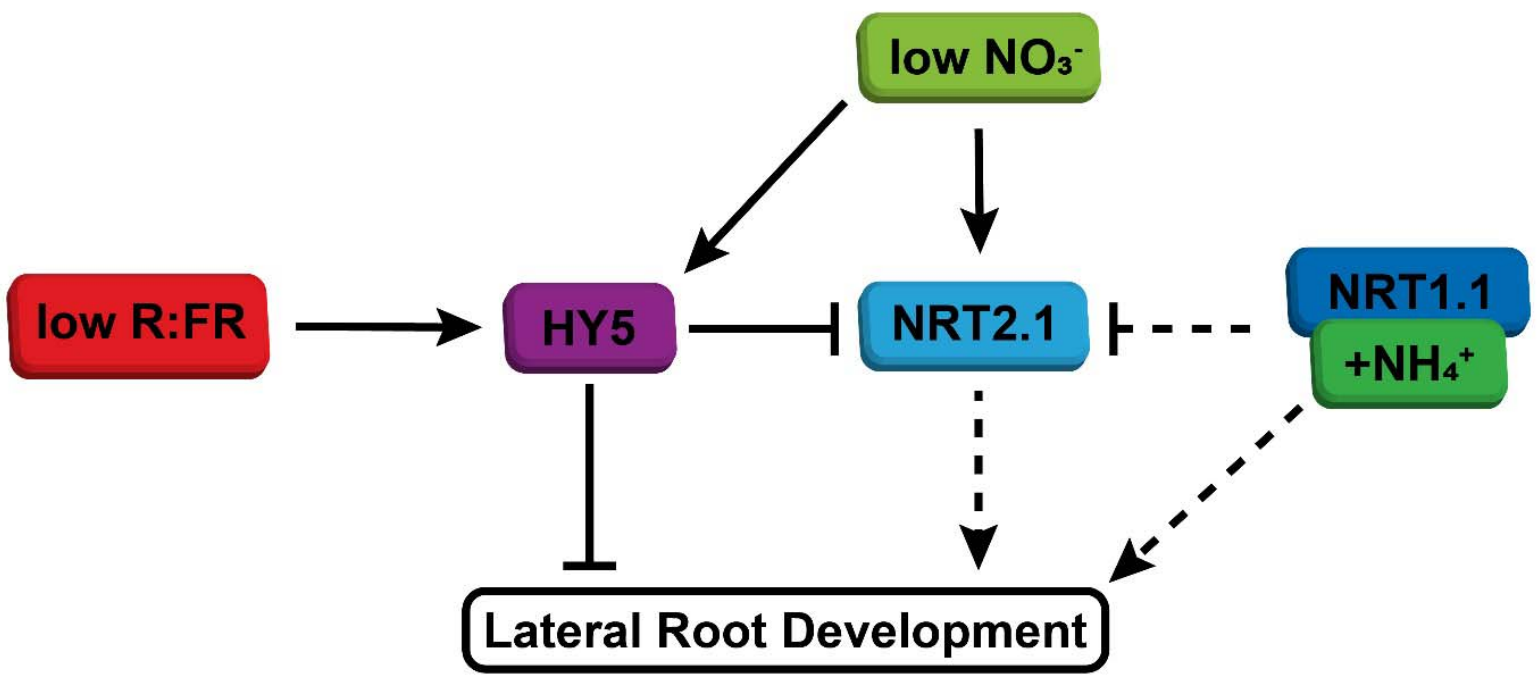

529 Figure 6: Simplified model of integration of shoot-perceived low R:FR and low nitrate

530 availability on lateral root development. WL+FR enhances HY5 expression, stabilization and

531 transport. In normal nitrate conditions this leads to reduced lateral root development, both due to

532 direct effect of HY5 on emergence, but also due to repression of NRT2.1, which has a positive effect

533 on lateral root development. When nitrate availability is low, both HY5 expression and NRT2.1

534 expression is enhanced, which results in further repression of lateral root development through HY5.

535 Ammonium, possibly via NRT1.1, bypasses low R:FR signaling through its direct effect on lateral

536 root development and also via its repression of NRT2.1 induction. 


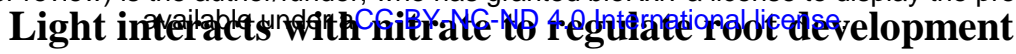

A

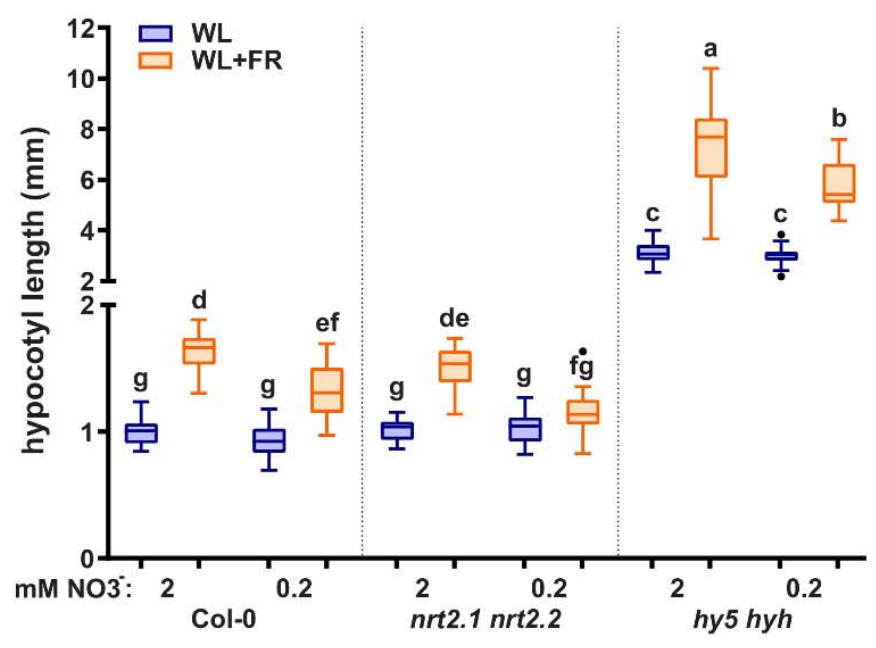

B

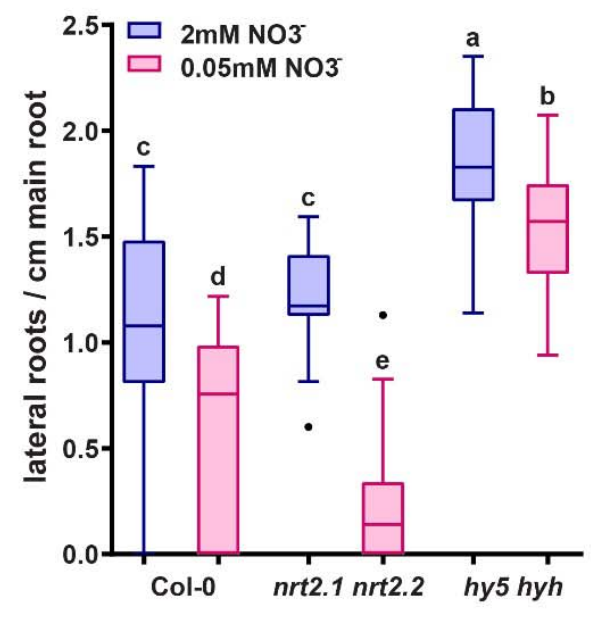

539 Figure S1. (A) Hypocotyl length of eight day old seedlings grown in WL on normal (2 mM) or low

$540(0.2 \mathrm{mM})$ nitrate. (B) Lateral root density of eight day old seedlings grown in WL on normal (2 $\mathrm{mM})$

541 or very low $(0.05 \mathrm{mM})$ nitrate. Letters denote statistically significant groups based on a mixed model

542 2-way ANOVA with posthoc tukey test $(\mathrm{p}<0.05)$. Scale bar $=1 \mathrm{~cm}$.

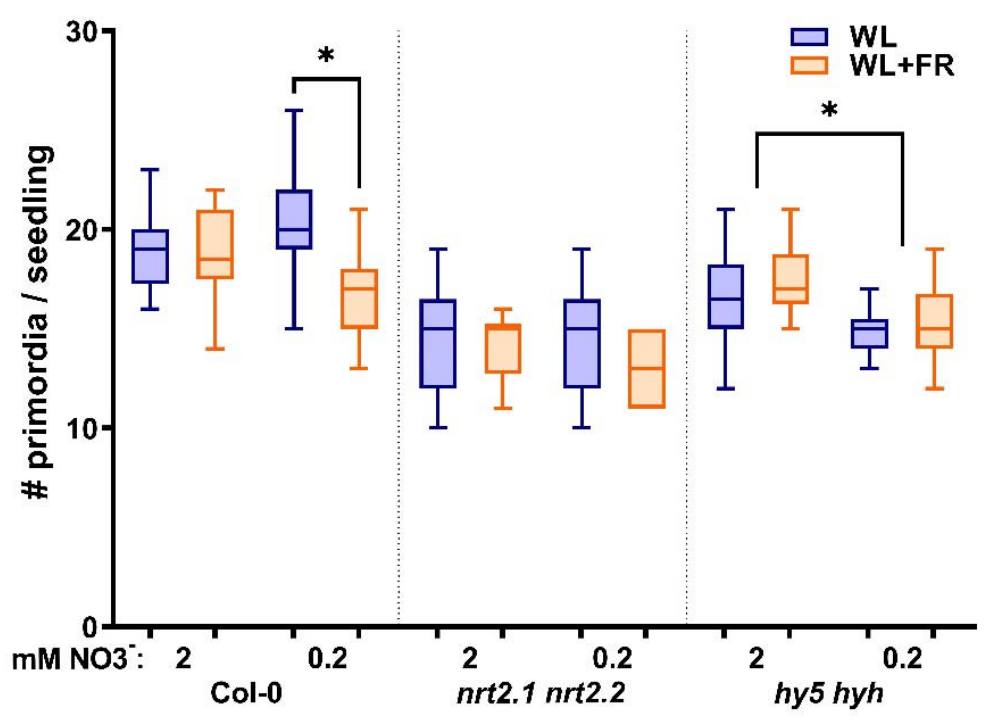

545 Figure S2. Total number of primordia per seedling from the experiment shown in figure 3. Statistics: mixed model 2-way ANOVA with post hoc Tukey test within the stage groups $(* \mathrm{p}<0.05)$. 


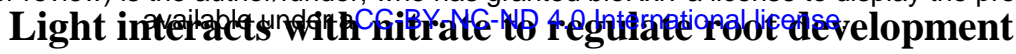

A

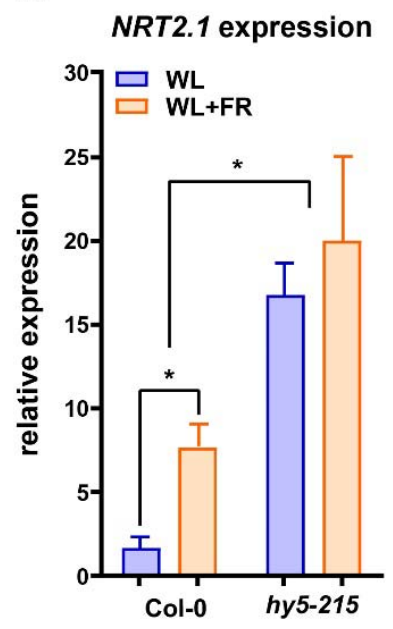

B

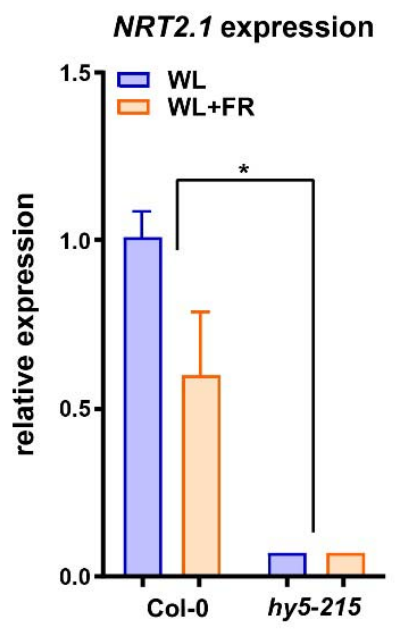

549 Figure S3. qPCR expression analysis of NRT2.1 using RNA of 5-day old seedling roots of Col-0 and 550 hy5-215 treated with combined WL or WL+FR, either on normal nitrate medium (A), or on $1 / 2$ MS 551 medium (B). ${ }^{*} \mathrm{p}<0.05$ with a one-way ANOVA plus post-hoc tukey test. 


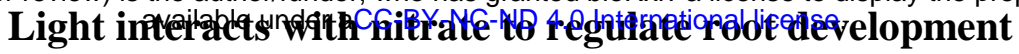

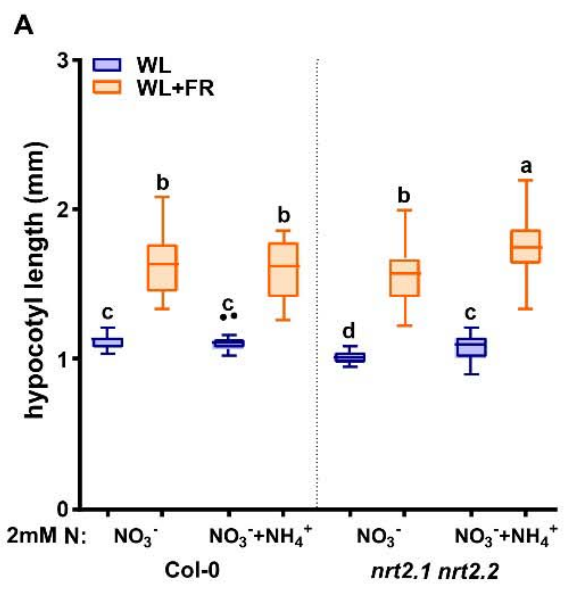

C

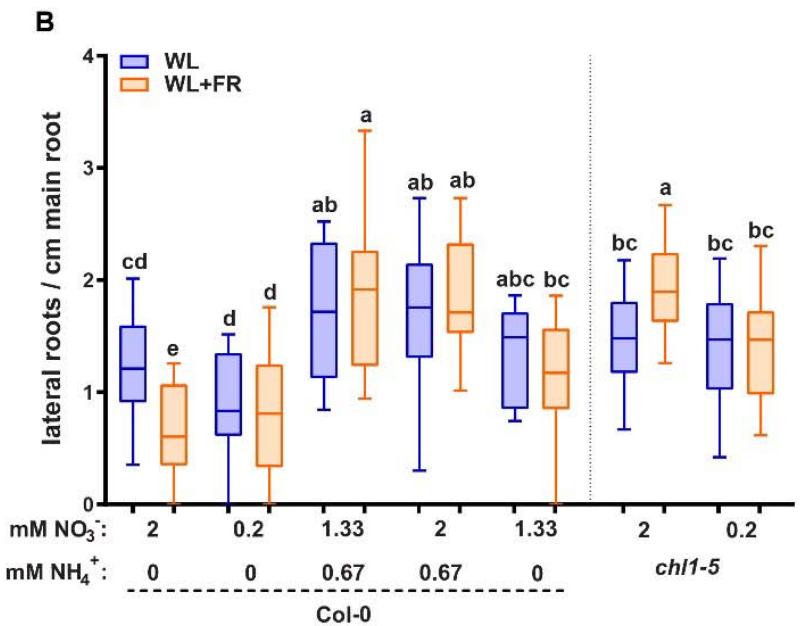

NRT1.1 expression

므 WL $W$ WR

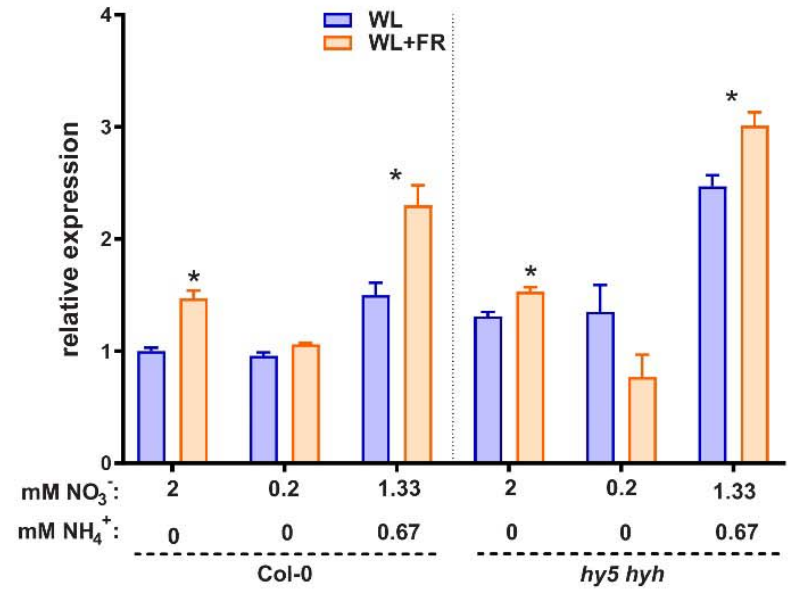

554 Figure S4. (A) Hypocotyl length of experiment shown in Figure 5. (B) Lateral root density of 8-day

555 old wild type Col-0 seedlings grown on normal nitrate $(2 \mathrm{mM})$, low $(02 \mathrm{mM})$, mixed nitrate

556 ammonium $\left(1.33 \mathrm{mM}+0.67 \mathrm{mM} \mathrm{NH}_{4}{ }^{+}\right)$, ammonium addition to normal nitrate $(2 \mathrm{mM}+0.67 \mathrm{mM}$

$\left.557 \mathrm{NH}_{4}{ }^{+}\right)$and $1.33 \mathrm{mM}$ nitrate. In the same experiment chll-5 (nrt1.1) was grown on normal (2 mM)

558 and low $(02 \mathrm{mM})$ nitrate. Letters denote statistically significant groups based on a mixed model 2-

559 way ANOVA with posthoc tukey test $(\mathrm{p}<0.05)$. (C) Expression analysis of NRT1.1 on the same

560 material as figure 4D-E ( $* \mathrm{p}<0.05$ vs $2 \mathrm{mM}$ Col-0 WL with a one-way ANOVA plus post-hoc tukey

561 test). 